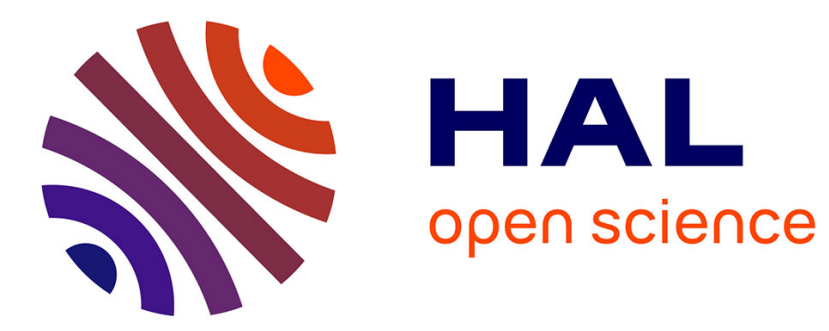

\title{
Molecular dynamics simulations of the effects of lipid oxidation on the permeability of cell membranes
}

Daniel Wiczew, Natalia Szulc, Mounir Tarek

\section{To cite this version:}

Daniel Wiczew, Natalia Szulc, Mounir Tarek. Molecular dynamics simulations of the effects of lipid oxidation on the permeability of cell membranes. Bioelectrochemistry, 2021, 141, pp.107869. 10.1016/j.bioelechem.2021.107869 . hal-03453851

\section{HAL Id: hal-03453851 \\ https://hal.univ-lorraine.fr/hal-03453851}

Submitted on 28 Nov 2021

HAL is a multi-disciplinary open access archive for the deposit and dissemination of scientific research documents, whether they are published or not. The documents may come from teaching and research institutions in France or abroad, or from public or private research centers.
L'archive ouverte pluridisciplinaire HAL, est destinée au dépôt et à la diffusion de documents scientifiques de niveau recherche, publiés ou non, émanant des établissements d'enseignement et de recherche français ou étrangers, des laboratoires publics ou privés.

\section{(ㅇ)(1) $\$$}

Distributed under a Creative Commons Attribution - NonCommercial - NoDerivatives 44.0 


\section{Bioelectrochemistry \\ Molecular dynamics simulations of the effects of lipid oxidation on the permeability of cell membranes \\ --Manuscript Draft--}

\begin{tabular}{|c|c|}
\hline Manuscript Number: & BIOELECHEM-D-20-00192R2 \\
\hline Section/Category: & Molecular and Cellular Electric Field Effects \\
\hline Keywords: & $\begin{array}{l}\text { Electroporation; secondary oxidation products; radical oxygen species; free energy } \\
\text { calculations; lipid bilayers; conductance }\end{array}$ \\
\hline First Author: & mounir tarek \\
\hline \multirow[t]{2}{*}{ Order of Authors: } & mounir tarek \\
\hline & Daniel Wiczew \\
\hline \multirow{2}{*}{ Suggested Reviewers: } & $\begin{array}{l}\text { Thomas Vernier } \\
\text { pvernier@odu.edu }\end{array}$ \\
\hline & $\begin{array}{l}\text { Andrei Pakhomov } \\
\text { apakhomo@odu.edu } \\
\text { Expert in Electroporation }\end{array}$ \\
\hline
\end{tabular}

Response to Reviewers: 


\title{
Molecular dynamic simulations of the effects of lipid
} oxidation on the permeability of cell membranes

\author{
Daniel Wiczew ${ }^{1,2 *}$, Natalia Szulc ${ }^{1,2}$, Mounir Tarek ${ }^{2^{*}}$ \\ ${ }^{1}$ Wroclaw University of Science and Technology, Department of Biomedical Engineering, \\ 50-370 Wroclaw, Poland, \\ ${ }^{2}$ Université de Lorraine, CNRS, LPCT, F-54000 Nancy, France \\ *Corresponding authors; \\ daniel.wiczew@pwr.edu.pl (D. Wiczew),_mounir.tarek@univ-lorraine.fr (M. Tarek)
}

\section{Highlights:}

1. The contribution of secondary oxidized lipids to permeabilization

2. Oxidized lipids form pores of several nanometers diameter in lipid bilayers

3. The lifetime of the formed pores depends on the membrane composition

4. A single pore accounts for the post-pulse change in cells' membrane conductance

5. Membrane domains with high oxidized lipids content are also highly permeable 


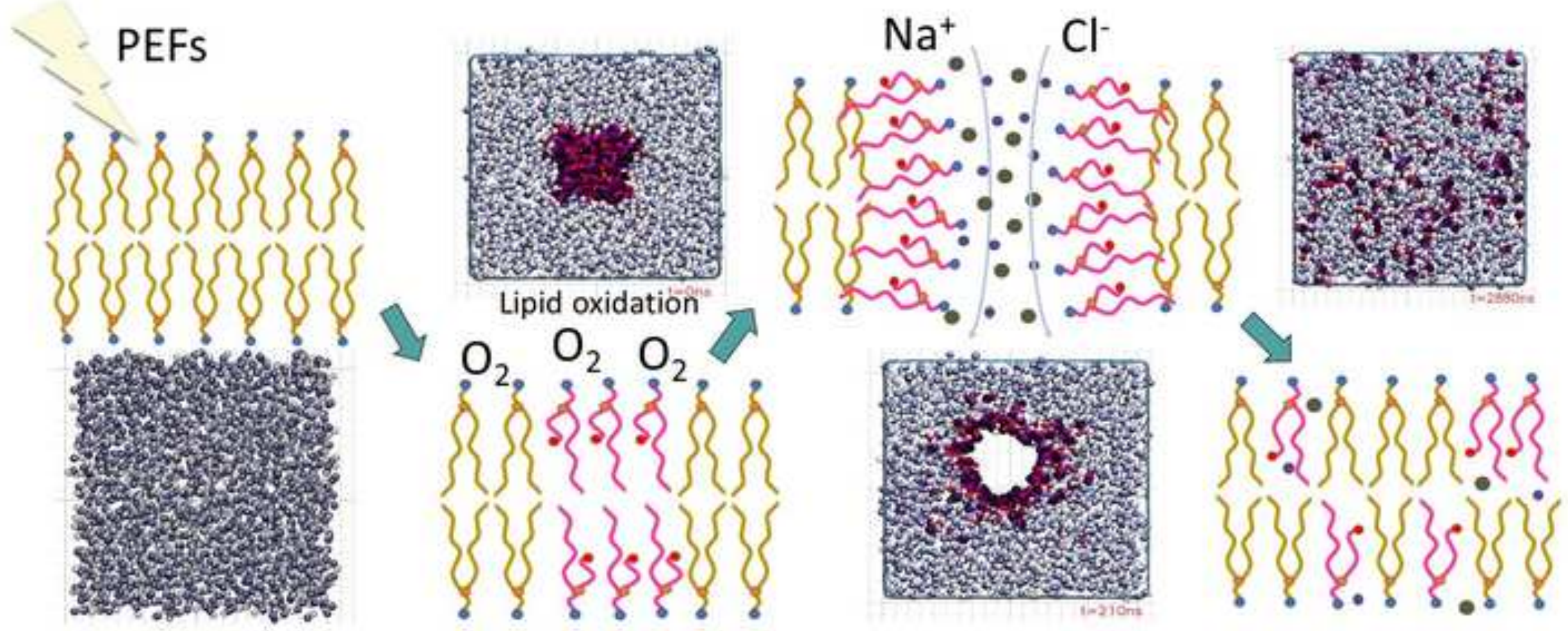




\title{
Molecular dynamics simulations of the effects of lipid oxidation on the permeability of cell membranes
}

\author{
Daniel Wiczew ${ }^{1,2 *}$, Natalia Szulc ${ }^{1,2}$, Mounir Tarek ${ }^{2 *}$ \\ ${ }^{1}$ Wroclaw University of Science and Technology, Department of Biomedical Engineering, \\ 50-370 Wroclaw, Poland, \\ ${ }^{2}$ Université de Lorraine, CNRS, LPCT, F-54000 Nancy, France
}

Provide full correspondence details here including e-mail for the:

*Corresponding authors;

E-mail addresses: daniel.wiczew@gmail.com (D. Wiczew), mounir.tarek@univ-lorraine.fr

(M. Tarek)

Keywords: electroporation, secondary oxidation products, radical oxygen species, free energy calculations, lipid bilayers, conductance

\begin{abstract}
The formation of transient pores in their membranes is a well-known mechanism of permeabilization of cells exposed to high-intensity electric pulses. However, the formation of such pores is not able to explain all aspects of the so-called electroporation phenomenon. In particular, the reasons for sustained permeability of cell membranes, persisting long after the pulses' application, remain elusive. The complete resealing of cell membranes takes indeed orders of magnitude longer than the time for electropore closure as reported from molecular dynamics (MD) investigations. Lipid peroxidation has been suggested as a possible mechanism to explain sustainable permeability of cell membranes. However, theoretical investigations of membrane lesions containing excess amounts of hydroperoxides have shown that the conductivities of such lesions were not high enough to account for the experimental measurements. Here, expanding on these studies, we investigate quantitatively the permeability of cell membrane lesions that underwent secondary oxidation. MD simulations and free energy calculations of lipid bilayers show that such lesions provide a better model of post-pulse permeable and conductive electropermeabilized cells. These results are further discussed in the context of sonoporation and ferroptosis, respectively a procedure and a phenomenon, among others, in which, alike electroporation, substantial lipid oxidation might be triggered.
\end{abstract}




\section{Introduction}

Electroporation (EP) is a well-known bio-method used to enhance the permeability of biological cell membranes [1-4]. This technique, which consists of exposing cells to pulsed electric fields (PEFs), enables the transport of various molecules such as drugs and genes, across their lipid membrane [5-8]. The current goal in improving our understanding of electroporation is the development of a comprehensive molecular-level description of the phenomenon. Information about the sequence of events describing electroporation is gathered from measurements of electrical currents through planar lipid bilayers along with the characterization of molecular transport of molecules into (or out of) cells subjected to electric field pulses and from atomistic level details provided by molecular dynamics (MD) simulations of model membranes. It is now well accepted that long and intense electrical pulses induce rearrangements of the membrane components (water and lipids), which ultimately lead to the formation of aqueous hydrophilic pores whose presence increases substantially the ionic and molecular transport through the otherwise impermeable lipid bilayers. One of the main unsolved questions related to the electroporation phenomenon is that the induced permeability of cell membranes can persist up to minutes after the applied electric fields are switched off [9-12]. This is in large discrepancy with the pores sustainability that MD simulations indicate, which rather close within a time scale in the order of tens to hundreds of ns $[13,14]$.

It has been suggested over two decades ago that membranes can be oxidized when subject to conditions similar to those of electroporation-based technologies. There is experimental evidence indeed that pulsed electric fields can increase the extent to which unsaturated lipid acyl chain peroxidation occurs. In particular, it has been shown that the application of external electric fields alters the phospholipid composition and properties of liposomes, vesicles, and cells [15-21]. The presence of oxidized lipids within bio-membranes is known to modify their physical properties and, especially their permeability. One cannot, therefore, exclude that molecular uptake following PEF treatments may, at least partially, take place through diffusion across oxidized/permeabilized lipid bilayer patches and not solely across the so-called electropores.

Several studies demonstrated that applying PEFs to cells induces generation of ROS and oxidative damage of unsaturated lipids, as confirmed for instance by the increased concentration of lipid oxidation products [4] and hydrogen peroxide [19,20]. PEFs enhance lipid peroxidation in bacteria [22,23], plant [24] and mammalian cells [25-27], as well as in liposomes made from polyunsaturated lipids [21,28,29]. Moreover, it has been shown that electric pulses can induce extracellular (electrochemical) [30,31] as well as intracellular [3135] ROS generation assumably due to destabilization of mitochondrial membranes. Both ROS concentration and the extent of lipid peroxidation increase with electric field intensity, pulse duration, and number of pulses $[17,18,35,36]$ and are correlated with cell membrane's permeability and cell death [16-18,32,37].

The most potent ROSs include the hydroxyl radical ( $\mathrm{HO} \bullet$ ), the superoxide radical anion $\left(\mathrm{O}_{2}^{-} \bullet\right)$, and the hydroperoxyl radical $(\mathrm{HOO} \bullet)$. These radicals are highly reactive and play a prominent role in the cells' lipid membrane oxidation [38]. They initiate chain oxidation reactions leading to oxidation of unsaturated lipids and thus change the physical properties of membranes $[39,40]$. When $\mathrm{HO}^{\bullet}$ and $\mathrm{HOO}^{\bullet}$ reach lipid membrane interiors containing 
unsaturated lipids $(\mathbf{L})$, they can initiate radical chain oxidation through an allylic hydrogen abstraction (see Schematic 1) leading to the formation of lipid radical $\mathbf{L} \cdot[40,41]$. The latter reacts with molecular oxygen, which is highly abundant in the membrane's interior [31] to form lipid peroxide radicals $\mathbf{L O O} \bullet$. This radical may further abstract hydrogen from another nearby lipid to form a primary peroxidation product, LOOH, (hydroperoxide), and a lipid radical $\mathbf{L} \bullet$, which becomes a substrate for yet another radical reaction. This reaction called propagation constitutes an ingredient for a chain reaction that takes place therefore between neighboring lipids and is terminated only by interaction with another radical or following removal of the radical from the lipid membrane system (e.g. by transferring the radical to a nearby water molecule) [42].

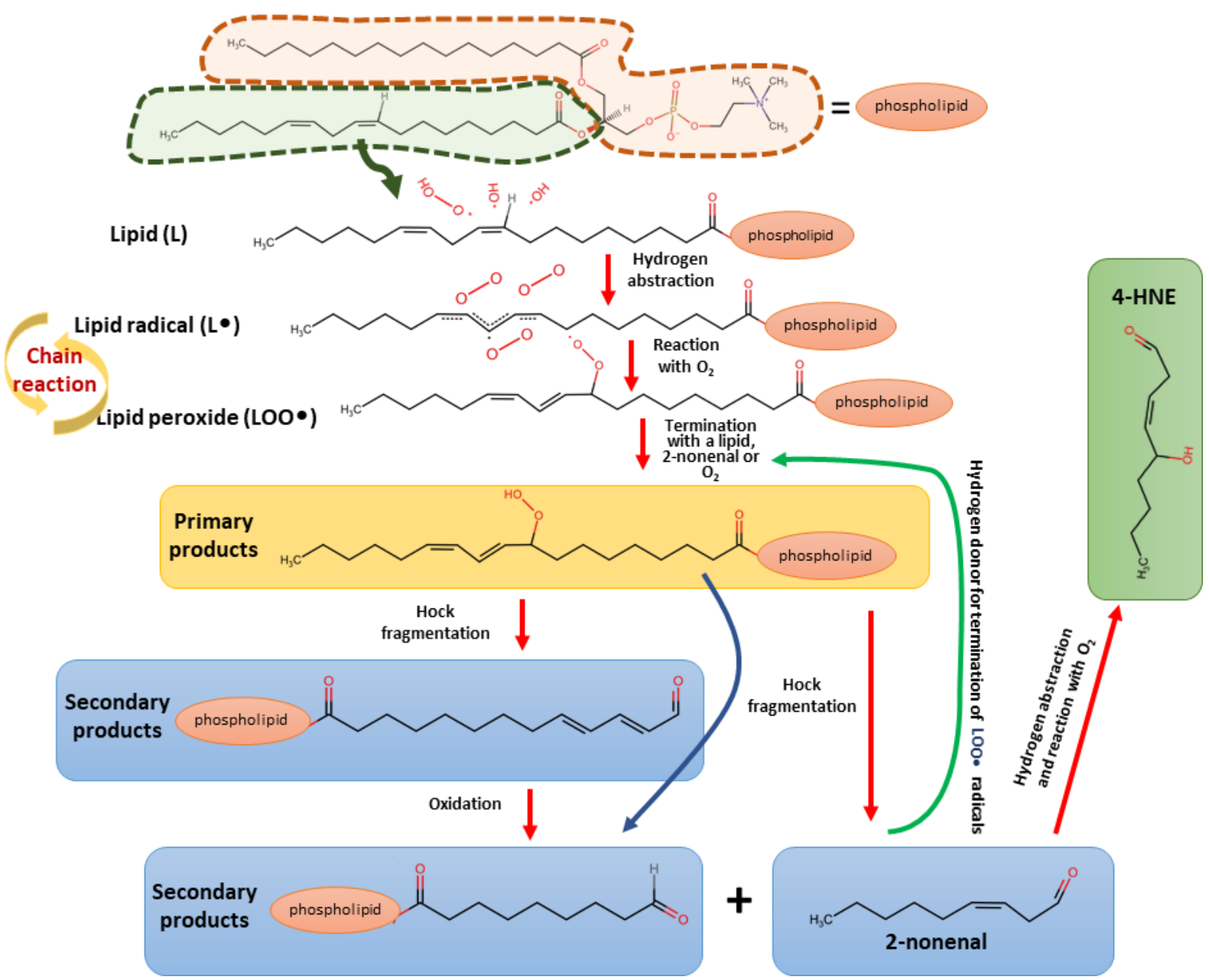

Schematic 1. Pathway for primary and secondary oxidation of a phospholipid (L) containing a single bis-allylic site e.g. linoleic acid, linoleate ester. The primary oxidation is a multistep process leading to the generation of hydroperoxides (and fuel the chain reaction - yellow arrows). The secondary oxidation can occur either directly through Hock fragmentation (blue arrow) with the formation of 2-nonenal [43] or through Hock fragmentation followed by oxidation as in the case of PoxnoPC [44,45]. 2-nonenal can be a hydrogen donor for LOO• (green arrow) and transfer the radical to another lipid initiating yet another chain reaction.

In a recent work [46] we investigated the permeability of lipid bilayers containing hydroperoxidized lipids mimicking therefore the presence of patches of lipids that underwent primary peroxidation chain reactions. Consistent with experiments on giant unilamellar 
vesicles (GUVs) containing up to $60 \%$ lipid hydroperoxides or exclusively lipid hydroperoxide species [47], our data showed that the bilayers preserved their membrane integrity. We estimated the lipid membrane conductance and permeability to monovalent ions using MD simulations and free energy calculations. The results were compared to available experimental measurements on electropermeabilized cells. Our data showed that the permeability and membrane conductance increase by several orders of magnitude in presence of excess amounts of hydroperoxidized lipid bilayers. However, this increase was not sufficient to reasonably account for the entire range of experimental measurements.

In this paper, we extend our investigation to study the permeability of membranes that underwent secondary oxidation. Indeed, oxidative lipid damage can result in various products with truncated lipid tails ending with either an aldehyde or carboxylic group [44,45]. Hydroperoxides are converted into secondary oxidation products through Hock fragmentation or Hock fragmentation followed by oxidation (see Schematic 1) [43]. Fast kinetic reaction ratios obtained from state-of-the-art quantum chemistry calculations suggest that primary oxidation product formation and degradation of primary to secondary oxidation products is faster than lateral diffusion of lipids [43]. Accordingly, our focus will be on the study of the properties and outcome of regions of highly oxidized lipids (called patches in this work).

Among the many lipids products of secondary oxidation, we have chosen 1-Hexadecanoyl-2(9-oxo-nonanoyl)-sn-glycero-3-phosphocholine (PoxnoPC) a stable byproduct of 1-palmitoyl2-linoleoyl-lecithin (PLPC) [48] or 1-palmitoyl-2-oleoyl-sn-glycero-3-phosphocholine (POPC) oxidation [49]. Previous MD simulations showed that oxidized lipids with an aldehyde group disturb indeed lipid bilayers more than the ones with a peroxide group [50,51]. PoxnoPC formation can results from direct oxidation of the corresponding hydoperoxide lipid or through formation first of alkenals and then oxidation, although, the latter reaction may be slower than the former since it requires an extra step [44,45]. The 2-nonenal is further converted to 4-HNE (4-hydroxynonenal) by reacting with a radical and oxygen in a similar fashion as unsaturated bonds in unsaturated lipids, thus potentially contributing to the lipid radical oxidation process (as a positive feedback loop - see green arrow in Schematic 1).

According to previous MD simulations, bilayers with aldehyde-truncated tails undergo spontaneous pore formation within a few hundred ns and lead in some cases to the bilayer complete disintegration (micellization) [50]. Furthermore, in light of experimental studies, Runas and Malmstadt [40] reported formation of pore defects in GUVs containing only $12.5 \%$ of PoxnoPC. Spontaneous pore formation in GUVs was also reported by Sankhagowit et al. [52] under conditions where aldehyde-truncated lipids were produced and their presence confirmed by $1 \mathrm{H}$ NMR. In a recent review Tsubone et al. [53] discussed lipid membrane remodelling due to photo-oxidation, describing in particular how oxidized lipids affect membrane permeability and how it may related to the formation of pores. The authors indicate that pore formation is linked to the presence of by-products of lipid oxidation rather than that of lipid hydroperoxides.

Our aim here is to thoroughly investigate the properties of lipid membranes that underwent such drastic chemical changes by considering lipid patches resulting from radical chain oxidation initiated by exposure to an electric field. We note that we will study the bilayers properties in absence of external field, modeling therefore the behavior of the system after the applied pulses have been switched off. We will specifically consider (Schematic 2) a model 
lipid bilayer composed of small bilayer patch of PoxnoPC representing the secondary oxidation product embedded in a pool of (POPC) bilayer representing the intact membrane focusing on their permeability.

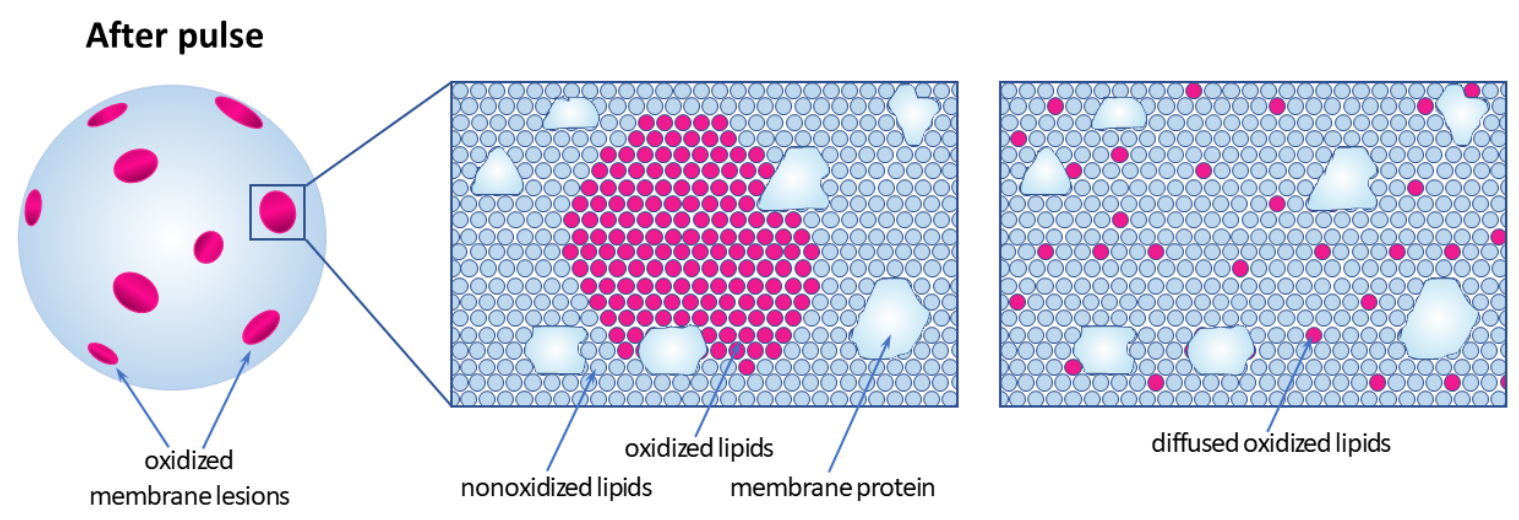

Schematic 2. Representation of oxidized membrane lesions (patches made of secondary oxidized lipids, drawn in purple) expected to be formed in the cell membrane following exposure to electric pulses (Left). The schematic is hypothetical, and the lesions are not drawn to scale. Middle: cartoon of molecular organization in one of the lesions, showing a patch of secondary oxidized lipids. Right: State of the membrane when the secondary oxidized lipids diffused out. Image adapted from [46].

To mimic molecular crowding in the cellular environment, and the scenario where the patches are created in a gel-like raft domains, we consider a second model membrane as well composed of a POPC bilayer with $40 \%$ cholesterol content. Both models (with and without cholesterol) contain a central bilayer patch of PoxnoPC. These simulations allow ones to directly follow for microseconds the features of such patches. Furthermore, similar to our previous investigation [46], we use free energy calculations (Unified Free Energy Dynamics (UFED) method [54]) to quantify the membrane conductance and permeability. We determine the free energy profiles of simple monovalent sodium and chloride ions diffusion through bilayers in which oxidized lipids have diffused out of the initial patch to form homogeneous domains (Schematic 2 right). These profiles will be used to determine the conductance of these model bilayers and the data was confronted with available experiments conducted on cells. Evidently our bilayer models do not embed the complexity of real cell membranes, but it is generally accepted that cells large conductance when subject of electroporation is due to leaks through its lipid membrane component.

\section{Materials and methods}

\subsection{Simulation of the model systems}

\subsubsection{Preparation of the model systems}

The initial system (SYST I) was built using PACKMOL and consisted of a large POPC bilayer (1152 lipids in total), in which the central patch was replaced by a PoxnoPC bilayer (64 molecules per leaflet) (SI, Table S2.1.1). PoxnoPC is a zwitterionic oxidized phospholipid bearing an aldehyde function at the end of its truncated sn-2 acyl chain. The whole bilayer model was further solvated in a solution containing sodium, calcium, and chloride ions (for 
composition details see SI, S2). The second system (SYST II) was analogous to the first one, but the POPC lipid bilayer contained a $40 \mathrm{~mol} \%$ of homogenously distributed cholesterol (SI, Table S2.1.1).

Additionally, four simple lipid bilayer systems (POPC) containing homogeneously distributed PoxnoPC at molar concentrations of $0 \%, 10 \%, 20 \%$, and $50 \%$ (respectively BIL1 to BIL4) were set and embedded in $\mathrm{NaCl}$ solutions (see SI, Table S3.1.1). Systems with the oxidized lipids (PoxnoPC) concentration of $80 \%$ or above were not stable (data are not shown here), therefore, we excluded this system from our simulations. BIL1 to BIL4 were studied to investigate how local concentrations of oxidized lipids after their diffusion out of the central patch (Schematic 2 right) affect the permeability and membrane conductance of the systems. BIL1 to BIL4 were built in a similar way to SYST I and SYST II using PACKMOL, except that PoxnoPC lipids were distributed randomly across the POPC lipid bilayer. The systems were run for over $1 \mu \mathrm{s}$ each to enable the extraction of the equilibrium features and properties.

\subsubsection{Simulations}

To perform the simulations, POPC and cholesterol were parameterized with the CHARMM36 lipid force field [55]. We developed a CHARMM-consistent Force field parameter for modeling PoxnoPC following the protocol used by Klauda et al. [55] using state-of-the-art coupled quantum chemistry calculations (see SI, S1). We used the TIP3P model [56] for water and the electronic continuum correction (ECC correction) for the ions representation [57] as it allows an accurate description of sodium and calcium ions interaction with the lipid bilayer's lipid headgroups (see also [46]).

The simulations were performed using the GROMACS 2019.2 package considering 3-d periodic boundary conditions, without any external electric field [58]. All systems were first minimized using the steepest descent algorithm to remove interatomic clashes, then equilibrated at constant temperature $\mathrm{T}(310 \mathrm{~K})$, using the Nose-Hoover thermostat $(\tau=1 \mathrm{ps})$ [59], and then simulated at constant (semi-isotropic) pressure P (1 atm), using the Parrinello-

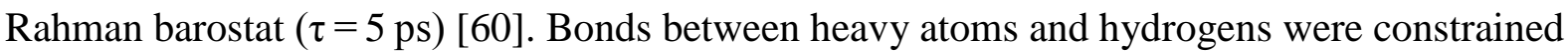
using the LINCS algorithm allowing the use of a 2 fs MD timestep. The long-range electrostatic interactions were evaluated using the Particle Mesh Ewald method [61]. The Fourier grid spacing for the Particle Mesh Ewald method was optimized at the beginning of each simulation, to get the highest performance during the simulation. A switching function was used between 0.8 and $1.2 \mathrm{~nm}$ to smoothly bring the short-range electrostatic interactions and the van der Waals interactions to 0 at $1.2 \mathrm{~nm}$.

\subsubsection{Lipid membranes MD analyses}

The bilayer thickness and the area per lipid (APL) of BIL1 to BIL4 were assessed using the PyBILT software [62]. To compare the influence of cholesterol on the lipid bilayer fluidity, D, the lateral diffusion coefficient of the PoxnoPC lipids was estimated using Stokes-Einstein's equation that links D to the mean square displacement (MSD) of molecules. For SYST I and SYST II, we considered ten PoxnoPC lipids not involved in the oxidized lipid patch or a pore (especially for SYST II, where the pore remains open). D was calculated using the gmx msd tool of GROMACS for the selected lipids considering the last $50 \mathrm{~ns}$ of the MD simulations. 
The sizes of the pores reported were estimated roughly by visual inspection using the grid projected onto the membrane with Visual Molecular Dynamics (VMD) [63] "grid" tool.

\subsection{Free energy calculations for BIL1 to BIL4, using the Unified Free Energy Dynamics (UFED)}

Free energy methods allow one to calculate free energy profiles as a function of a collective variable $(\mathrm{CV})$. The $\mathrm{CV}$ maps a high dimensional coordinate of an explored process (conformational change, ion permeation) into a low dimensional representation, that can be manipulated to obtain desired results. In this study, we used the UFED approach $[64,65]$. The latter combines ideas from two methods: d-AFED (driven Adiabatic Free Energy Molecular Dynamics) and metadynamics, resulting in superior convergence in comparison to both methods. UFED was successfully used in our previous works [46],[65],[66] to obtain the potential of mean force (PMF) profile of ions and small radical species along the normal to a lipid bilayer. d-AFED works by connecting a $\mathrm{CV} q$ with harmonic potential (with coupling constant $\boldsymbol{\kappa}$ ) to an extended variable $s$ (see equation (1)). The extended variable is adiabatically decoupled from the rest of the system, which is achieved by choosing a large $\mathbf{\kappa}$ and a mass $\mathbf{m}$ of the extended variable. Such treatment allows the extended variable $s$ to be set to a temperature $T_{s}$ higher than that of the system to ensure better sampling of states in the phase space. The temperature $T_{s}$ is usually set such that $\mathrm{k}_{\mathrm{b}} \mathrm{T}$ ( $\mathrm{k}_{\mathrm{b}}$ is Boltzmann constant) is similar to the energetic barrier of the studied process. Thus, the extended variable can easily cross the barrier and drags the CV alongside. In UFED, the regions of the phase space are explored using a bias that ensures a better sampling of the infrequently explored phase space regions. The bias, similar to the one used in metadynamics is in the form of Gaussian hills with given height $h$ and variance sigma, which is deposited gradually along the extended variable trajectory. The PMF $\Phi(s)$ profile can be recovered by numerically integrating from the force $F(s)$ acting on the extended variable $s$.

$$
\begin{aligned}
& F(s)=-\frac{\delta \Phi(s)}{\delta s}=\boldsymbol{\kappa}(q-s)_{s} \\
& \Phi(s)=-\int_{S_{\text {min }}}^{s_{\text {max }}} F(s) d s
\end{aligned}
$$

where $s$ and $q$ are extended variable and collective variable respectively and $\boldsymbol{\kappa}$ denotes the coupling constant for the mentioned harmonic potential. In our study, the extended variable was defined as the center of the lipid bilayer, whereas the $\mathrm{CV}$ was defined as the z-position of selected ion (here sodium or chloride) with respect to the bilayer center.

\subsubsection{System preparation of BILI and BIL4 for UFED}

The bilayer thickness and area per lipid were calculated to verify convergence of the equilibration. The electrostatic potential profile along the membrane normal (z-axis) was evaluated considering the last $100 \mathrm{~ns}$ of the simulation using the 1D Poisson's equation,

$$
\phi(z)=-\varepsilon_{0}^{-1} \iint \rho\left(z^{\prime \prime}\right) d z^{\prime \prime} d z^{\prime}
$$


where $\rho(z)$ is the molecular charge distribution along $z$. The integration was performed by slicing the system into 1000 bins and averaging charge density within each bin and then integrating it according to the above equation using the g-potential tool of GROMACS.

\subsubsection{UFED parameters}

The aim was to obtain the PMF profiles of the translocation of an ion (sodium or chloride) along the direction normal to the BIL1, BIL2, BIL3 and BIL4 lipid bilayers. Therefore, the collective variable $(\mathrm{CV})$ was defined as a $\mathrm{z}$-position of the ion. In agreement with our previous investigation [46], this CV was coupled with a harmonic constant of $10^{4} \mathrm{~kJ} / \mathrm{mol} / \mathrm{nm}$ to a metavariable (extended variable $\mathbf{s}$ ) of mass $\mathrm{m}_{\mathrm{s}}=2 \cdot 10^{4} \mathrm{a} . \mathrm{m}$.u. The extended system was coupled to a bath at a temperature $\mathrm{T}_{\mathrm{s}}=400 \mathrm{~K}$ using a generalized Gaussian moment thermostat (GGMT). Gaussian-shaped potential hills, with a height of $1.0 \mathrm{~kJ} / \mathrm{mol}$ and width of $0.01 \mathrm{~nm}$, were deposited every $5000 \mathrm{MD}$ steps (100 fs). To prevent an overall movement of the lipid bilayer in the $\mathrm{z}$-direction, the center of mass of the system (estimated from the average $\mathrm{z}$ position of the Lipids head group phosphorus atoms) was restrained in the z-position using a harmonic potential with a spring constant of $3500 \mathrm{~kJ} \cdot \mathrm{mol}^{-1} \mathrm{~nm}^{-2}$.

\subsubsection{UFED simulation protocol for BIL1 to BILA}

The free energy calculations were performed using GROMACS 4.6 .3 [67] patched with PLUMED 1.3 [68] that includes the implementation of d-AFED/UFED free-energy calculations $[54,64]$. The parameters for the MD were similar to the system preparation phase (2.2.1 above), except for the LINCS algorithm [69] parameters, which were adjusted toward more accurate calculations ("lincs-order" set to 6 and "lincs-iter" set to 2). The temperature of the simulation was set $\mathrm{T}=300 \mathrm{~K}$ both for steering and UFED calculations (see paragraph below), and thermostat coupling constants were set to 1.6 ps. More details about these parameters can be found in the SI, section S3.4.

A multiple walkers' strategy, which involves the parallel evolution of multiple (sixteen) metadynamics simulations sharing the same bias potential history file, was employed in order to increase computational efficiency. The UFED calculations were hence performed using 16 separate sub-simulations (called further walkers) in parallel. Each walker started at a different initial configuration, in which the positions of ions $(\mathrm{Na}$ or $\mathrm{Cl}$ ) along the bilayer normal was obtained by steering the latter toward the center of the lipid bilayer in an independent MD run. The initial configuration of this "steered dynamics" run was the last frame from the well equilibrated $(1 \mu \mathrm{s})$ trajectory described in section 2.2.1. The steering was performed using a spring constant of $3500 \mathrm{~kJ} . \mathrm{mol}^{-1} \mathrm{~nm}^{-2}$ and a velocity of $0.3 \mathrm{~nm} / \mathrm{ns}$ using PLUMED 1.3. The MD parameters for steering were the same as for UFED, the same goes for temperature that was set to $\mathrm{T}=300 \mathrm{~K} .16$ configurations were selected among the ones saved every $0.1 \mathrm{~nm}$, from 6.5 $\mathrm{nm}$ to $3.4 \mathrm{~nm}$ to the center of the lipid bilayer ( 32 configurations in total). The other 16 were used for a second run to check convergence and estimate uncertainty.

The UFED calculations were performed for $80 \mathrm{~ns}$ for each walker (16 walkers for each calculation) of the systems listed in the 2.2.1 (8 calculations in total - 4 for chloride, BIL1BIL4, 4 for sodium, BIL1-BIL4). The output for the UFED variables (q, s, etc.) was saved 
every 0.04 ps of the simulations. An example of two trajectories for two walkers is shown in the SI, Figure S3.4.1.

\subsubsection{Analysis of PMF profiles for BIL1 to BIL4}

The PMF profiles were evaluated using equation (3) together with custom Matlab scripts (Matlab R2019a, MathWorks) which were adapted from the scripts developed by Cuendet et al. $[46,54,65]$. This was achieved by a multistep calculation workflow that started by dividing obtained force along a collective variable $s$ into 120 bins in the interval $\mathrm{z}=[-3.0,0.0] \mathrm{nm}$. Then the forces $F=\kappa(q-s)_{s}$ were sampled over the UFED run and averaged within each bin. These forces then were slightly smoothed with a kernel smoothing regression (Matlab function $\mathrm{ksr}$ ) with a bandwidth of $1 / 30 \mathrm{~nm}$. Finally, the forces were integrated with cumulative trapezoidal numerical integration (Matlab function cumtrapz) to obtain the PMF over the interval $\mathrm{z}=[-3.0,0.0] \mathrm{nm}$. This profile was then mirrored across the center of the bilayer.

As stated above, to estimate the uncertainty of the profiles, we performed an additional UFED run for each of the investigated systems (4 UFED calculations for chloride and 4 for sodium as in the last paragraph of 2.2.3). The second UFED run was performed in the same way as the first run, except that the initial configurations of the walkers were different. The PMF profiles were determined the same way as the previous run. The averaged PMF profiles are shown in Fig. 3. The two runs are used to check the convergence and reliability of the UFED method see S3.5.2 in the SI.

\subsection{Estimations of the permeability and the membrane conductance for BILI to BILA}

The bilayer permeability to a given ion was calculated according to the inhomogeneous solubility-diffusion model as in (4).

$$
P=\left(\int_{z_{1}}^{z_{2}} \frac{\exp \left(\Phi_{i o n}(z) / R T\right.}{D_{\text {ion }}(z)} d z\right)^{-1}
$$

where $\Phi_{\text {ion }}(z)$ is the PMF ( $\mathrm{Na}$ or $\mathrm{Cl}$ ) ion along the z-axis, $R$ the universal gas constant, $T$ the temperature of the system, and $D_{\text {ion }}(z)$ the diffusion coefficient of the ion along the $\mathrm{z}$-axis (see next section). The integration boundaries were taken as $z \in[-3.0,0.0] \mathrm{nm}$. The uncertainty of the values was estimated given the uncertainty of the PMF and that on the $D_{\text {ion }}(z)$. The former was estimated based on the maximum and minimum value of obtained permeation from two separate UFED runs. The lower and upper two values were multiplied by 1.33 and 0.67 , corresponding to the maximum variation between maximum/minimum values relative to smoothed $D_{\text {ion }}(z)$ profile (more in the SI 4.1). The uncertainty is about $+/-33 \%$ in the average scenario (highest error among all diffusion calculations). It was applied to all other ion diffusion calculations across the bilayer normal.

As in [46], the total and ionic membrane conductances (in $\mathrm{S} / \mathrm{m}^{2}$ ) of $\mathrm{Na}$ and $\mathrm{Cl}$ were calculated using [70,71]:

$$
G_{m}=G_{N a}+G_{C l}=\frac{N_{A}\left|q_{e}\right|^{2}}{k_{B} T} c\left(P_{N a}+P_{C l}\right)
$$


where $G_{m} G_{N a}$ and $G_{C l}$ are respectively the total sodium and chloride membrane conductances. $N_{A}$ the Avogadro's number, $q_{e}$ the ion's charge, $k_{B}$ and $T$ the Boltzmann's constant and the temperature. The ionic concentration and $\mathrm{T}$ for this work were $50 \mathrm{mM}$ and $\mathrm{T}=300 \mathrm{~K}$ respectively - the same as used in the UFED and diffusion calculations. The uncertainties were calculated based on the uncertainty propagation rule for addition, diffusion's uncertainty, and two UFED calculations, obtaining lower and upper bound for membrane conductance in a similar manner as in the case of permeation.

\subsection{Diffusion coefficients for monovalent ions across lipid membrane}

The diffusion coefficients of the ions across the BIL1 to BIL4 membranes used to estimate the permeability of the ions were determined similarly as in [46],[70] by performing umbrella sampling on $(\mathrm{Na}$ or $\mathrm{Cl})$ ions constrained in the z-positions using a harmonic potential with a spring constant $\kappa=3500 \mathrm{~kJ} . \mathrm{mol}^{-1} \mathrm{~nm}^{-2}$. The initial configurations were obtained from steered trajectories performed during preparation to the UFED calculations (see 2.2.3 above), thus giving 32 configurations in total. The MD parameters were the same as in the UFED calculations (see section 2.2.1). For each walker $i \in[0,31]$, a $10 \mathrm{~ns}$ long trajectory was generated, where the first two ns were considered as an equilibration phase. The remaining 8 ns were divided into four equal segments. For each one, the diffusion coefficient for a given configuration $i$ was calculated as:

$$
D_{i}=\frac{\left.<\delta z^{2}\right\rangle_{i}}{\tau_{i}}
$$

The variable $\tau_{i}$ here is the correlation time for the $\mathrm{i}$-th configuration and was calculated using the method of Hummer [72]. Further, $D_{i}$ obtained for each of the configurations was averaged over the four $2 \mathrm{~ns}$ long parts. Finally, the averaged values were smoothed with a kernel smoothing regression (Matlab function ksr) with a bandwidth of $0.2 \mathrm{~nm}$. The smoothed profiles were used in the calculation of membrane permeability and conductance (see SI, Figure S4.1).

\section{Results}

\subsection{Patches of secondary oxidation lipids undergo spontaneous and long-lived pore formation}

Fig. 1 reports snapshots from the MD trajectories of SYST I and SYST II. The systems represent domains of (respectively pure POPC and POPC: 40\% cholesterol) including small patches of PoxnoPC located in the center of domain representing "hot spots" resulting from a peroxidation chain reaction initiated by a ROS attack, followed by secondary oxidation of the hydroperoxide lipids (see Schematic 1). 


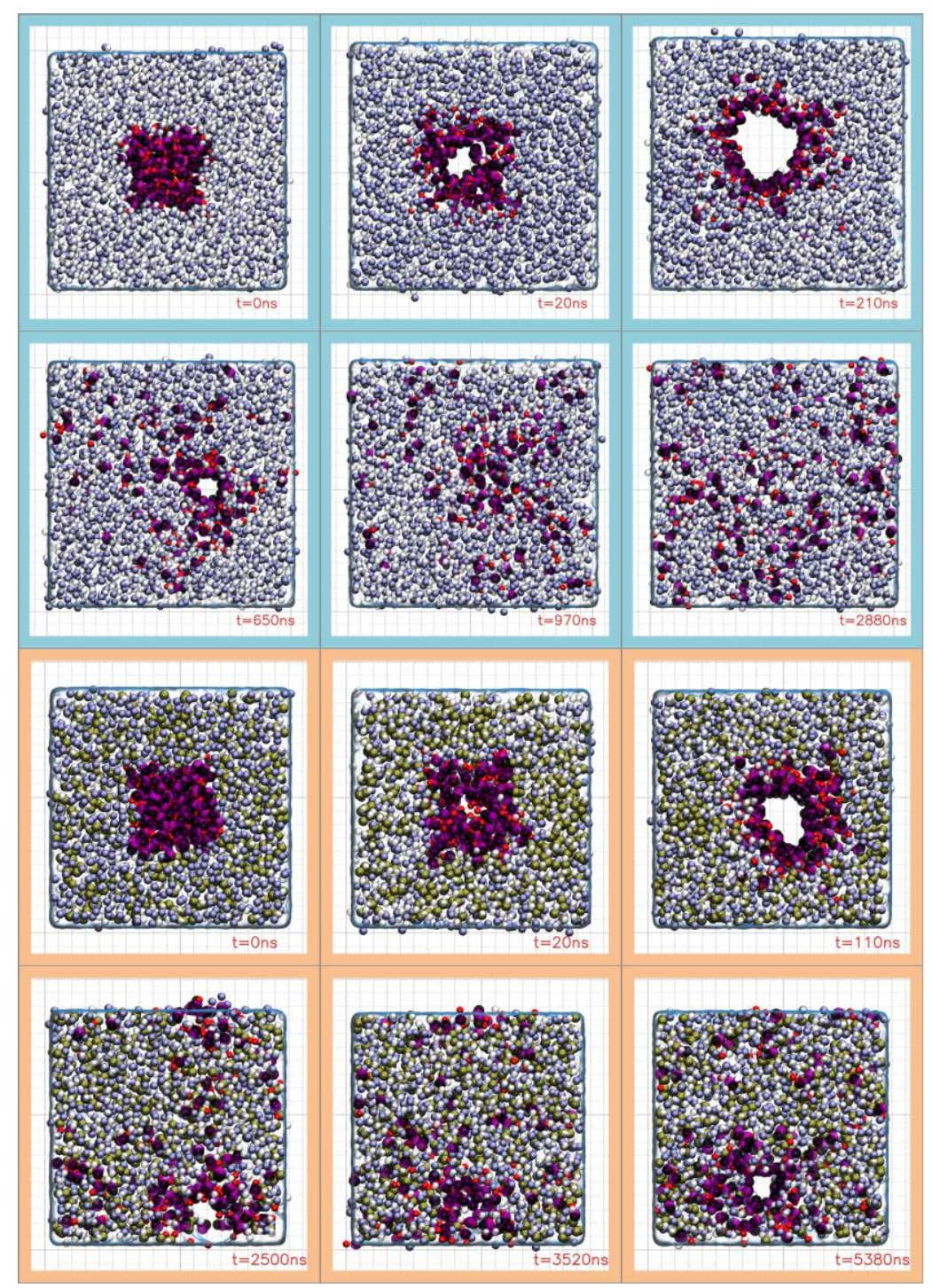

Fig. 1. Time evolution of pore formation and its closure in the bilayer systems without cholesterol (SYST I : top six blue panels) and with cholesterol (SYST II: bottom six orange panels). The grid size is $1 \mathrm{~nm}$. The blue and white spheres represent the choline and phosphate groups of the POPC molecules' zwitterionic head groups. The magenta and black spheres represent the choline and phosphate groups of PoxnoPCs head groups, the red spheres correspond to aldehyde oxygen of PoxnoPCs lipid tails, and the cholesterol's $\mathrm{OH}$ groups are drawn as yellow spheres. The corresponding membrane cross-sections can be found in SI, Figure S2.3.1. and Figure S2.3.2. along with the videos of the full MD trajectories.

The simulations (without any consideration of external electric field) indicate that the presence of such a PoxnoPC patch, given the localized high concentration of lipid aldehydes, leads within a few tens of nanoseconds to a pore formation. As the simulations are carried out at 0 surface tension (semi-isotropic NPT ensemble at 1 atm, see Casciola \& Tarek [7]), these pores expand quickly to reach a maximum size of $\sim 7 \mathrm{~nm}$ and $5 \mathrm{~nm}$ in diameter for SYST I and SYST II respectively. Such pores are therefore large enough to enables the transport of RNA and DNA [73,74] strands and fluorescent dyes such as: Yo-Pro, Propidium Iodide, or Calcein. For SYST I, as the surrounding lipids (POPC) is in the liquid crystalline state, PoxnoPC molecules diffuse laterally out of the formed pore during the $\sim 3 \mu \mathrm{s}$ MD simulation (Fig 1. $6^{\text {th }}$ snapshot in the blue panels), leading to a pore shrinking, basically as the number of PoxnoPC molecules 
forming the pore decreased. Within less than $1 \mu \mathrm{s}$, this pore shrinks hence to a diameter of $~$ $0.6 \mathrm{~nm}$, then the pore closes after about $1 \mu \mathrm{s}$.

For SYST II, the diffusion of PoxnoPC molecules out of the initial patch was much less rapid, due to the gel-like dynamics of the surrounding lipid bilayer (POPOC $+40 \%$ cholesterol). Toward the end of the MD run (over $5 \mu \mathrm{s}$ ) the pore did not completely close and the number of oxidized lipids around the pore remains high enough to maintain an aperture of $\sim 1.35 \mathrm{~nm}$ diameter. Estimation of the lateral diffusion coefficients D of PoxnoPC in this (6.2 \pm 0.9$) \times$ $10^{-12} \mathrm{~m}^{2} \mathrm{~s}^{-1}$, revealed a twofold decrease compared to D in the pure POPC bilayer, namely $(12.0 \pm 2.8) \times 10^{-12} \mathrm{~m}^{2} \mathrm{~s}^{-1}$. This twofold decrease of lateral diffusion of PoxnoPC due to cholesterol inclusion, observed also in other simulations [75,76], explains the reason for sustained pore life time in SYST II compared to SYST I.

\subsection{Properties of the bilayers with homogeneously distributed PoxnoPC lipids}

In order to study the permeability of regions of cell membranes that contain oxidized lesions long after the initial state (see Schematic $2 \mathrm{c}$ ), we have modeled several systems for which PoxnoPC have diffused enough so that the pores close (see for instance last panel Fig. 1 of SYST I). The systems BIL1 to BIL4 model such membrane domains, in which the distribution of PoxnoPC lipids in a POPC bilayers with concentrations ranging from $10 \%$ to $50 \%$ is set homogenous.

First, MD runs exceeding 100 ns for every system studied did not reveal any destabilization of the membranes at these oxidized lipids concentrations. No pores/water or ion leaks were formed during the simulations. The structural properties of the bilayers reported in Fig. 2 shows monotonic changes of the area per lipid and of the bilayer thickness as percentage of oxidized lipids increases. These trends were similar to those reported by Vernier et al. [25], namely higher percentage of oxidized lipids result in higher area per lipid and lower membrane thickness. Additionally, the bilayer thickness and the area per lipid for the pure POPC bilayers (respectively $3.93 \mathrm{~nm}$ and $0.65 \mathrm{~nm}^{2}$ ) are within $1 \%$ of the reported experimental values at 30C which provides some confidence in the force field parameters and MD protocols used herein.
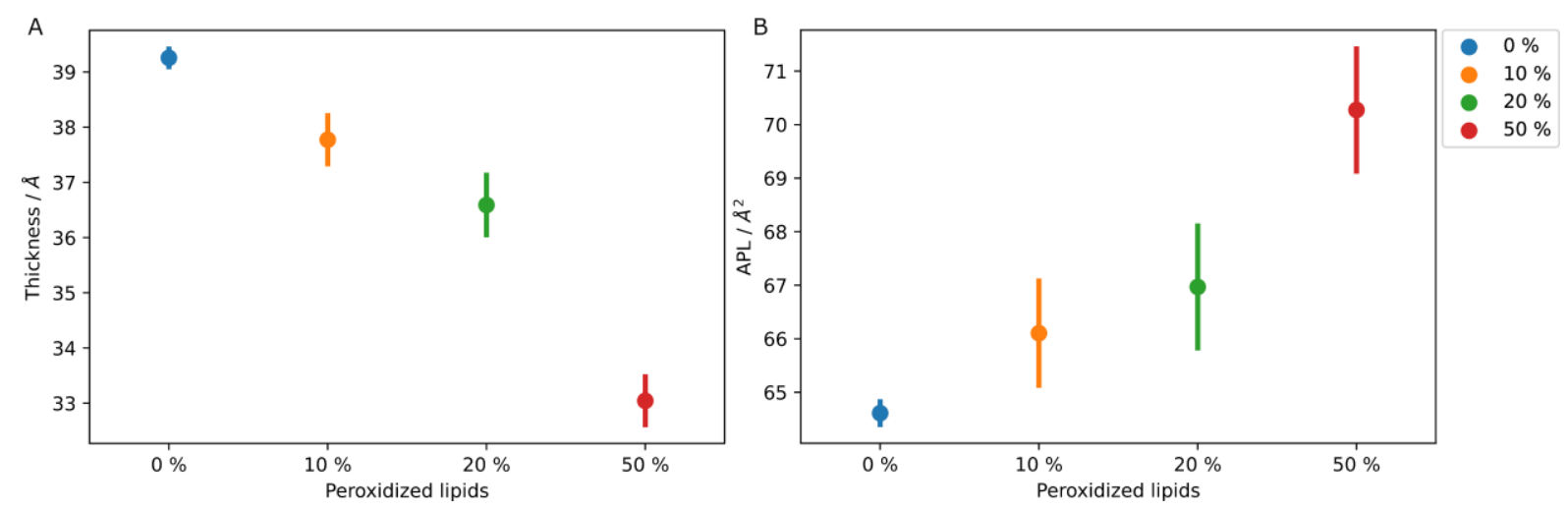

Fig. 2. Thickness (A) and average area per lipid (B) for BIL1, BIL2, BIL3, BIL4 lipid membranes. The legend (red $-50 \%$, green $-20 \%$, orange $-10 \%$, blue $-0 \%$ ) reports the percentage of secondary oxidized lipids (PoxnoPC) in POPC bilayer, where $0 \%$ represents data for the pure POPC bilayer. The bars indicate standard deviations based on the last $100 \mathrm{~ns}$ of the MD trajectories. 


\subsection{Ion penetration barrier across the bilayer with homogeneously distributed PoxnoPC lipids}

The PMF profiles of the translocation of $\mathrm{Na}^{+}$and $\mathrm{Cl}^{-}$across the bilayer systems (BIL1, BIL2, BIL3, BIL4) with 0\% (pure POPC bilayer) 10\%, 20\%, and 50\% homogeneous distribution of PoxnoPC are reported in Fig. 3. All the profiles are $\Lambda$-shaped, similar to those reported in previous studies of ion permeation through the membrane containing lipid hydroperoxides. As the percentage of PoxnoPC increases, the free energy barrier progressively decreases for both $\mathrm{Na}^{+}$and $\mathrm{Cl}^{-}$, similarly as reported for ions across lipids bilayers containing hydroperoxides or for water permeation across lipids bilayers containing oxidized lipids. As expected, the bilayer with 50\% PoxnoPC lipids presents the lowest barrier to ion permeation.

Here, the calculations with concentrations higher than $50 \%$ of oxidized lipids were not considered due to the formation of pores in the systems (for instance at $80 \%$ PoxnoPC), as was the case for SYST I and SYST II. It is fair to assume accordingly that bilayer domains with oxidized lipid concentration higher than $50 \%$ would have negligible or virtually zero free energy barrier to permeation as ions can pass freely through the formed pores.
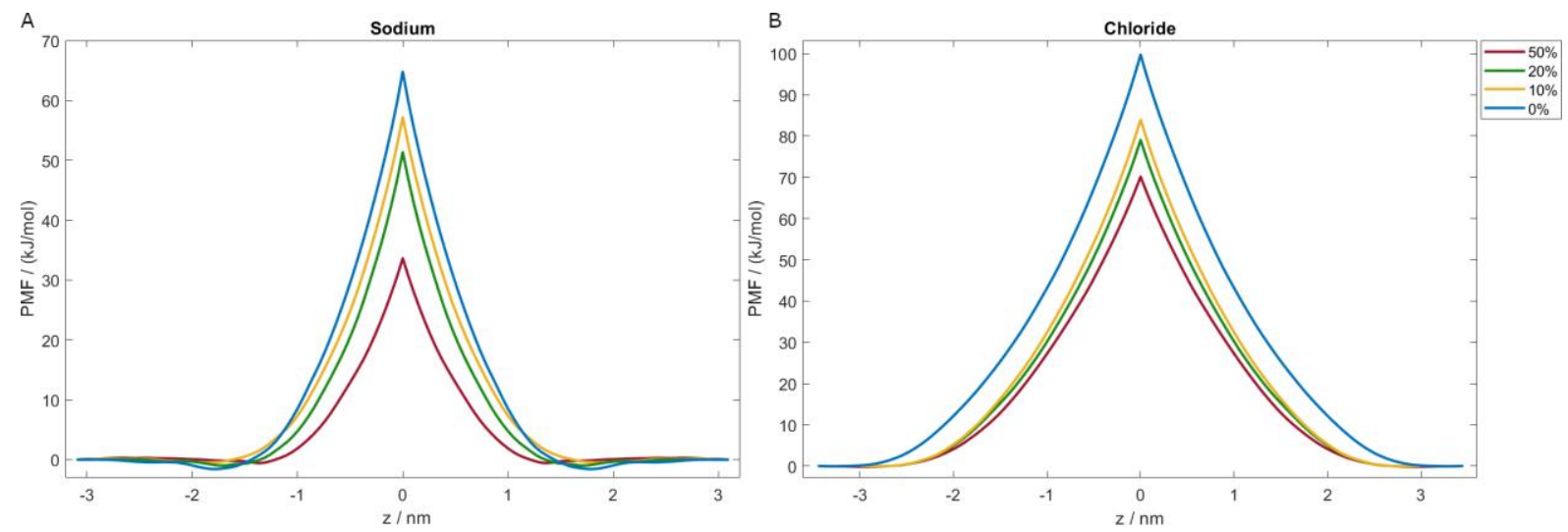

Fig. 3. The PMF profiles of $\mathrm{Na}^{+}(\mathrm{A})$ and $\mathrm{Cl}^{-}(\mathrm{B})$ in BILL1 to BILL4. The legend represents the ration PoxnoPC:POPC and $\mathrm{z}$ the ion position with respect to the center of the bilayer (located at $\mathrm{z}=0$ ). The error bars on the free energy estimates (see 2.2.3) are less than $2 \mathrm{~kJ} / \mathrm{mol}$ (see $\mathrm{S} 3.5$ for details).

\subsection{Permeability and ion conductance of lipid bilayer with secondary oxidation products}

The permeability to each ion species and the total membrane conductance (sum of $\mathrm{Na}^{+}$and $\mathrm{Cl}^{-}$ membrane conductance) of BIL1 to BIL4 are depicted in Fig. 4. As the percentage of PoxnoPC (secondary oxidation lipids) increases, the membrane permeability, and conductance do so as well. Noticeably, the $\mathrm{Na}^{+}$permeability of the bilayer with $50 \%$ PoxnoPC is 5 orders of magnitude higher (increase from $10-11 \mathrm{~m} / \mathrm{s}$ to $3.10-6 \mathrm{~m} / \mathrm{s}$ ) than the permeability of the pure POPC bilayer. The same holds for $\mathrm{Cl}^{-}$, where the permeability increases from $2.10^{-15} \mathrm{~m} / \mathrm{s}$ to $6.10^{-11} \mathrm{~m} / \mathrm{s}$, i.e. by over 4 orders of magnitude.

These changes are reflected in the magnitude of changes in the total membrane conductance. Hence, the analysis of the simulation data using eq (6) shows that the latter is 5 orders of magnitude higher for the bilayer with 50\% PoxnoPC relative to that of the non-oxidized lipid bilayer (pure POPC). 

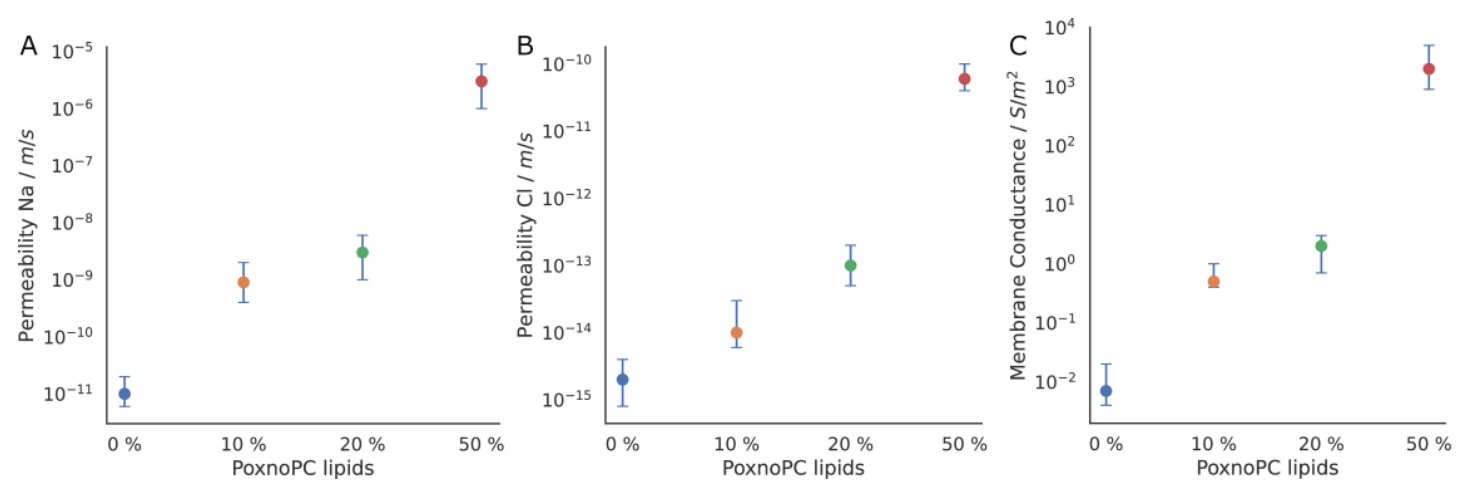

Fig. 4. Permeability to $\mathrm{Na}^{+}(\mathrm{A})$ and $\mathrm{Cl}^{-}(\mathrm{B})$ and overall membrane for both ions (C) of the BIL1 - BIL4 defined by the percentage of secondary oxidized lipids (PoxnoPC) in the POPC bilayer, ( $0 \%$, corresponding to BIL1 is the pure POPC lipid membrane.

\section{Discussion}

It has been suggested in recent studies that lipid oxidation could be a possible reason for sustained cell membrane permeability. This phenomenon manifests itself as persistence of permeability following treatment with a high intensity electric field that lasts up to minutes after the electric fields that trigger the so-called membrane electroporation is switched off. As we will discuss later, this phenomenon may not only be encountered in electroporation [77] but also whenever cells or tissues are exposed to high levels of ROS, e.g. subject to ultrasounds triggering sonoporation [65] or to photodynamic therapy [78].

To date, however, no rationale for this phenomenon has been offered. Previous studies by our group have concluded that membranes that underwent primary oxidation alone, namely membranes with an excess amount of hydroperoxides, were not leaky enough to account for cell permeability and conductance measured in experiments where cells were exposed to PEF usually used in electroporation-based technologies and treatments [46]. The aim of the present study was to investigate whether oxidative lesions of cell membranes composed of secondary lipid oxidation products can give rise to such behavior.

Hence, we have herein conducted MD simulations of molecular systems aiming at modeling the behavior of cell membranes subjected to lipid oxidation. The first set of simulations was meant to model membranes immediately after exposure to the electric field and therefore to oxidation of the lipid membranes by ROS. We have assumed that peroxidation of lipids is followed by quick secondary oxidation, due to its fast kinetic of radical chemistry as discussed in the introduction. Peroxidation of the lipids being a chain reaction [79], it would produce "spots" or "patches" of oxidized lipids at the lipid bilayer domains of the surface of a cell membrane (Schematic 2 - left panel) [72,78,79]. We have studied the outcome of such spots when they are formed in a fluid lipid phase, namely a POPC lipid bilayer above its phase transition temperature, or when formed instead in a "gel-like" domain modeled here as a POPC bilayer containing a high amount of cholesterol.

\subsection{Secondary oxidation lipids and pore formation in the lipid bilayer}

The results of Section 3.1 show that a small patch of secondary oxidation aldehyde lipids (PoxnoPC) is enough to form spontaneously a large pore (up to $\sim 7 \mathrm{~nm}$ diameter) in SYST I 
and SYST II. This indicates that the oxidation of only a small area of a cell membrane would induce formation of wide enough pores to transport ions and large molecules like RNA, DNA and fluorescent dyes. This is consistent with other MD investigations where a high concentration of various aldehydes in lipids bilayers induced pore formation and micellization $[50,80]$. Pore formation is also consistent with various experimental reports. Hence, In an study of giant unilamellar vesicles (GUVs), Runas \& Malmstadt reported that $12.5 \%$ or higher concentration of PoxnoPC induces fluorescein-dextran uptake and suspected that it could be due to pore formation [40]. Tsubone et al. discussed as well lipid membrane remodelling due to photo-oxidation, describing in particular how oxidized lipids affect membrane permeability and how it may related to pores forming in presence of oxidized lipids with oxidized shortened alkyl chain (like PoxnoPC) [53]. Sankhagowit et al. [52] reported that GUVs with light-induced lipid oxidation show line tensions changes and surface area per lipid changes, that could be interpreted as a pore opening.

\subsection{Secondary oxidation lipid's pores and their conductance in comparison to GH3 cells}

While commonly described electric field-induced pores (also called electropores) following rearrangement of the membrane components are easily attributed to the membrane damage allowing ionic and molecular transport by computational [25] and experimental studies [81] they do not explain sustained (long-time scale) post-pulse permeation.

Among the experimental methods which enable the detection of lipid oxidation products one may cite spectrophotometric [82,83], chromatographic [84], and immunochemical [85] ones. Other methods using fluorescent dyes are widely applied as well to probe oxidation in live cells [86]. In a recent work, Michel et al. introduced a novel method for the detection of oxidation by ratiometric fluorescence measurements of BODIPY-C11 dye using total internal reflection fluorescence (TIRF) microscopy, enabling therefore to single out the cell membrane oxidation [87]. They used this method to assess lipid peroxidation in CHO-K1 cells subjected to the action of pulsed electric fields. Their investigation led to conclude that lipid oxidation can indeed occur at the plasma membrane when the cells are subject to electric pulses, but this was not retained as the cause of long-lasting membrane permeabilization as measured by Yo-Pro uptake. A we will show below a small number of oxidized patches, probably below the detection limit of methods such as the one used by Michel et al. are enough to enhance substantially the permeation to ion, dyes. We recall that the pores crated by each of these patches (see section 3.1) are several nanometers large, i.e. wide enough to transport molecules such as Yo-Pro, Propidium Iodide, dyes often used to probe membrane permeability.

Hence, in the following, we will question, first and foremost, if pores that are formed by oxidative patches similar to those studied above can be, in anyway, correlated to the sustained permeability and conductance measured in cells exposed to PEFs. We recall from [46] that $N_{\text {pore }}$ the number of pores needed in a cell to provide an increase of normalized membrane conductance $\Delta g$ (in units $\mathrm{nS} / \mathrm{pF}$ ) can be expressed as:

$$
N_{\text {pore }}=\frac{\Delta g C_{\text {cell }}}{G_{\text {pore }}}
$$

where $C_{c e l l}$ is the total capacitance of the cell membrane and $G_{\text {pore }}$ the pore's conductance. The analytical expression for cylindrical pore's conductance [in $\mathrm{nS}]$ is expressed [88] as: 


$$
G_{\text {pore }}=\frac{2 \pi \sigma_{\text {pore }} r^{2} \text { pore }}{\pi r_{\text {pore }}+2 d_{\text {pore }}}
$$

where $\sigma_{\text {pore }}=\left(\sigma_{\text {extra }}-\sigma_{\text {intra }}\right) / \ln \left(\sigma_{\text {extra }} / \sigma_{\text {intra }}\right)$, is the effective conductivity, a function of $\sigma_{\text {extra }}$ and $\sigma_{\text {intra }}$ the extra- and intracellular conductivities, $r_{\text {pore }}$ is the diameter of the pore and $d_{\text {pore }}$ the pore's length through the lipid membrane. More details about these calculations can be found in [46] (section 4.3).

Using equation (7) and (8) one can therefore estimate the number of pores required to reach a given change in cell's conductance $\Delta g$ (e.g. assuming N_pores $=1$ ), so the change $\Delta g$ per pore $\left(\Delta g / N_{\text {pore }}\right)$

Hence, considering $r_{\text {pore }} \sim 2.5 \mathrm{~nm}$ (data from section 3.1) and $C_{\text {cell }} \sim 6 p F$ [89], $\sigma_{\text {extra }}=$ $1.48 \mathrm{~S} / \mathrm{m}$ [90] and $\sigma_{\text {intra }}=0.5 \mathrm{~S} / \mathrm{m}$ extracted from experiment, and $d_{\text {pore }} \sim 5 \mathrm{~nm}$, the thickness of the bilayer, we find that $\Delta g / N_{\text {pore }}$ amounts to $\sim 0.3 \mathrm{nS} / \mathrm{pF}$ per pore. Quite interestingly, the conductance changes reported for GH3 cells measured 5 to $10 \mathrm{~s}$ after the application of $60 \mathrm{~ns}$ PEFs (with amplitudes ranging from 4.8 to $14.6 \mathrm{kV} / \mathrm{cm}$ ) is in the range of $0.1-1.5 \mathrm{nS} / \mathrm{pF}$ (values obtained from Fig 3a. of [91] normalized with the GH3 cell's capacitance of $6 \mathrm{pF}[89])$.

Hence, our data indicate clearly that one to few pores of the size of those formed by the small patch of oxidized lipids in our work ( $\sim 0.3 \mathrm{nS} / \mathrm{pF}$ per pore) are enough to account for the changes reported in membrane's conductance in the GH3 cells (between $0.1-1.5 \mathrm{nS} / \mathrm{pF}$ ). The mere lipid patch of 6.6 x $6.6 \mathrm{~nm}$ of 64 truncated oxidized PoxnoPC lipids per leaflet mimicking the presence of patches that underwent oxidation chain reactions, is able -in absence of applies electric field- to form a pore large enough to explain most of the change in ion conductance, and probably permeation to other large molecules.

\subsection{Pore lifetime in a gel like lipid bilayer models}

In the above discussion, we highlighted that, pores formed by small patches of oxidized lipids could explain the high permeability of cells. However, the timescale during which pores forming in our simple model of fluid-like bilayers (SYST I) remain open is too short (less than one microsecond) to account for all experimental observation. To mimic crowded conditions in the cellular environment, and the scenario where these patches are created in a gel-like raft domain of the cell membrane, we considered enriching lipid domains composed of highly fluid lipids (like POPC) with cholesterol, decreasing thereby their fluidity [92]. We set up a similar lipid bilayer as previously, but with the addition of $40 \mathrm{~mol} \%$ cholesterol (SYST II). As stated in Section 3.1, the oxidized lipids of the patch quickly form pores and under these conditions, the lifetime of the pore increased significantly, from $\sim 1 \mu$ s to over $5 \mu \mathrm{s}$. Our data does not allow to determine an upper bound for the pore lifetime. Nevertheless, it shows that the membrane fluidity and the location of the "oxidative spots" might significantly affect pore's lifespan.

Consideration of cholesterol is a simple approximation to the molecular crowding in the real cell membranes in which $\sim 50 \%$ of the cell's surface is composed of proteins [93]. The latter have a strong negative influence on the lipid membrane's fluidity [94-96], hindering drastically the diffusion of oxidized species. We can therefore safely conclude based on our investigations that the presence of secondary oxidation lipid patches resulting from direct oxidation could 
form pores with lifetimes far longer than herein assessed from our simulations (or from biophysical experiments on vesicles) where proteins are absent.

Further in cells, the repair of such oxidized lipid patches may be a long-time process and may imply various complex biological mechanisms. For instance, in Chinese hamster ovary (CHO) cells, it takes $\sim 4 \mathrm{~h}$ to remove $11 \%$ content of the secondary oxidation product PazePC (1palmitoyl-2-azelaoyl-sn-glycero-3-phosphocholine) via a catabolic pathway involving the Phospholipase $\mathrm{PLA}_{2}$ [97]. Peroxiredoxin 6 (Prdx6) a bifunctional enzyme with GSH peroxidase and phospholipase A2 (PLA2) activities associated with oxidative damage repair in cell membranes has been shown to decrease the content of phospholipid hydroperoxides in in pulmonary microvascular endothelial cells to control levels within a couple of hours [98]. It is unclear whether oxidative damage repair is faster or slower for primary versus secondary oxidation products. Furthermore, the increased levels of reactive oxygen species (ROS) has also been shown to impair oxidized lipid removal due to cell starvation, preventing them from resealing completely as pointed out previously [30]. Regardless of the processes of damage repair, the latter is nonetheless occurring on time scales order of magnitudes larger than those required for physical closure of pores formed with chemically unaffected lipids, reported from modelling studies to be in order of tens of nanoseconds.

\subsection{Membrane conductance after truncated oxidized lipid diffusion out of the patch and pore closure}

The simulations of SYST I and SYST II show that oxidized lipids (PoxnoPC) form clusters, after they diffuse out of the pore. These concentrations decrease with time (due to further lateral diffusion of PoxnoPC) while the domains enlarge. In order to study the permeability of such domains, we estimated the conductance (see Section 3.4) for model (POPC) membranes with $50 \%, 20 \%, 10 \%$ PoxnoPC in comparison with experimental values of conductance normalized to the GH3 cell's $6 \mathrm{pF}$ capacity. We first calculated the normalized conductance of the 3 systems using the capacitance $\mathrm{C}_{\mathrm{m}}$ per unit area $\left(\sim 1 \mu \mathrm{F} / \mathrm{cm}^{2}\right)$ which resulted in $200 \mathrm{nS} / \mathrm{pF}, 0.2 \mathrm{nS} / \mathrm{pF}$, $0.05 \mathrm{nS} / \mathrm{pF}$. (for more information see [46]).

Quite interestingly, the modeled conductance increase $\Delta g_{o x}$ is comparable to the conductance increase $\Delta g_{\text {exp }}$ reported for GH6 cells and recorded 2-3 min after subject them to a $60 \mathrm{~ns}$ single $\mathrm{PEF}$, namely $\sim 0.05 \mathrm{nS} / \mathrm{pF}$ to $\sim 0.5 \mathrm{nS} / \mathrm{pF}$ with respect to control. We can estimate the fraction $f_{o x}$ of the cell's membrane that is needed to obtain the measured conductance (more details and derivation in [46], section 4.1) using:

$$
f_{\text {ox }}=\frac{A_{o x}}{A_{\text {cell }}}=\frac{\Delta g_{\text {exp }}}{G_{o x} / C_{m}}=\frac{\Delta g_{\text {exp }}}{\Delta g_{o x}}
$$

Accordingly, agreement with the data reported for GH6 cells would require that about $0.025 \%$ to $0.25 \%$ of the cell's lipids membrane area to be oxidized at $50 \%$, whereas for the other limiting case with $10 \%$ degree of oxidation, it would require $100 \%$ to $1000 \%$ of the lipid membrane area to be oxidized.

Since pore re-forming was observed during the simulation with excess amounts (over $80 \%$ ) of homogeneous PoxnoPC distribution, it could be hypothesized, that a mixture of pores and regions with a high concentration of oxidized lipids could also lead to significant cell's sustained permeation. 


\subsection{Lipid oxidation and pore formation beyond electroporation}

In the introduction of this paper, we have indicated that evidence suggests that PEFs do not generate ROS directly, but appear to enhance their intracellular or extracellular generation [3234]. The scenarios presented above are therefore not restricted to electroporation-based treatments. They might be encountered whenever ROS generation is enhanced, in which case one would expect sustainable pore formation due to secondary lipid oxidation. Most obviously, one should expect similar pore occurrences when cells and tissues are subject to photodynamic therapy (PDT) or cold atmospheric plasma (CAP)-based therapy [99-102]. PDT is a therapeutic modality in which photosensitizers excited at specific wavelengths generate ROS [103]. As a result, the target cells loaded with such photosensitizers undergo damage that can lead up to necrosis or apoptosis partly due to PDT-induced lipid oxidation and its consequences on membrane permeability (see [104] for a recent review).

In the present discussion, one would like to focus yet on another biomedical technique besides electroporation or PDT, known to enhance lipid oxidation, namely sonoporation. In recent years, research in the field of microbubble (MB)-assisted ultrasound (also known as sonoporation or sonopermeabilization) has gained momentum. The technique aims at delivering therapeutic molecules including nucleic acids, anti-cancer drugs, peptides and antibodies in vitro and in vivo. It has been known for some time that ultrasound disturbs the dynamic balance of the intracellular ROS, suggesting that the physical damage from sonoporation causes an increase in intracellular calcium which induces a rise of ROS in mitochondria. The exogenous molecular uptake and the concentration of intracellular ROS were quantified at the cellular level to evaluate the extent of the cell response to sonoporation. The results suggest that that intracellular ROS generation in reversible sonoporated cells can impact their long-term fate.

Recently we have shown, using combined MD simulations and in vitro experiments, that under certain sonoporation conditions, ROS can form inside the microbubbles [65]. Experimental data confirmed theoretical predictions that MBs favour indeed the spontaneous formation of a host of free radicals in which the hydroxyl radical $(\mathrm{HO} \bullet)$ was the main species produced upon US exposure. The study has shown that these radicals could easily diffuse through the MB shell toward the surrounding aqueous phase and reach the nearby cells' membranes. An increase of both intracellular and extracellular ROS concentrations that might stem from the application of the sonoporation technique is shown to impact the permeability of the exposed cell. The exact mechanism is not fully described though one often assumes that cell membranes with extensive oxidation damage become leaky. Along the lines of previous simulations showing that $\mathrm{HO} \cdot$ can penetrate deep in the hydrophobic core of lipids (POPC bilayers [38])[65] to reach the peroxidation sites [105], namely their bis-allylic hydrogen atoms. Analyses using ROS scavengers and inhibitors suggested that the ROS produced under sonoporation conditions using MB-assisted ultrasound plays a role in the permeabilization of cell plasma membranes and the in vitro delivery of plasmid DNA [65]. The extent of permeability of cells to large molecules when subject to sonoporation is likely due (at least in part) to pores formed by secondary oxidation products, similar to those revealed in this study. 
Lipid oxidation is also implicated in a variety of other complex biological processes. Programmed cell death is a process that leads a living cell into controllable death that in contrast to necrosis, does not involve leakage of organelles neither induces proinflammatory response [106]. In recent years, many other forms of cell death aside from apoptosis were discovered, e.g. necroptosis or ferroptosis. The latter relates to an iron-dependent mechanism involving its accumulation [107]. Ferroptosis is characterized by intensive ROS generation, lipid oxidation, NAPDH oxidation, and GSH depletion [108,109]. Studies support a general model of cell disruption in necroptosis through the formation of small pores of a few nanometres radius in the plasma membrane [107,110]. It was reported that cells can be protected from ferroptosis by "pore blockage" using polyethylene glycol (PEG 1450 and 3350), but not sucrose or raffinose [107] suggesting that the pores are larger than $1 \mathrm{~nm}$ and smaller than $2.4 \mathrm{~nm}$ in diameter. Pedrera et al. reported that ferroptosis progression involves the production of oxidized lipids prior to a sustained increase of cytosolic $\mathrm{Ca}^{2+}$ and final plasma membrane breakdown. As high lipid peroxidation is the most dramatic effect detected in ferroptosis, one could infer, consistently with the present work, that the pores detected are formed by local concentrations of secondary lipid oxidation [111]. Such pores' formation in the cell plasma membranes can cause a significant drop in the transmembrane potential due to the influx of calcium, sodium, chloride ions, and outflux of potassium ions. Such changes in the ionic balance, might cause a depolarization of the cell and the activation of Voltage-Gated Calcium Channels (VGCC), and therefore the further influx of calcium.

\section{Conclusions}

In summary, we investigated in this study whether secondary lipid oxidation products could contribute to the long-lived permeability and conductance to the electropermeabilized cell lipid membranes, which persists after the application of electric pulses used in Electroporation based technologies and treatments. We performed large-scale (a model of 1152 all-atom lipids) simulations that show that long lifetime nanometer pores form due to the presence of a small patch of oxidized lipids resulting from radical oxidation chain reactions. We estimated the permeability of such pores and compared it with available measurements in cells. We investigated the influence of molecular crowding on the lifetime of such pores. Finally, we studied as well the permeability of domains corresponding to cell membrane surfaces in which oxidized lipids have diffused out of the patches (long time process) but presence of which still influences the conductance properties of the bilayer.

Overall, our modeling results and data analyses indicate that the pore formation due to the presence of secondary oxidation aldehyde lipids can quantitatively match the lowest to the highest experimentally reported conductance changes in cells that underwent PEF treatment. These pores live far longer than electropores that result from reorganization of the lipid components under the influence of high electric fields.

We have shown that the composition of the lipid bilayers significantly affects the lifetime of the pores. Free energy calculations allowed one to show that lipid membrane containing excess amounts secondary oxidation products of remains leaky even after the pore closure. Along with our previous report [46] this study therefore presents molecular level quantitative analysis of 
the permeation of cell membranes subject to oxidative damage confronted with available experimental measurements.

The present results rely on the initial hypothesis that the oxidized lipids are generated as patches of lipids. This hypothesis, while consistent with the fact that (i) peroxidation of highly unsaturated lipids triggered by species such as the highly potent hydroxyl radical $\mathrm{HO} \bullet$ is a chain reaction, and (ii) that lipids are packed in a membrane to favor its propagation is yet to be confirmed. Experimentally detection of such nanometer-scale domains in cell membranes or in model systems such as vesicles is hardly achievable. Computational chemistry methods could in principle be used to model these chain reactions in a realistic (model bilayer) environment, providing therefore support of such hypothesis. Indeed, quantum mechanics/molecular mechanics (QM/MM) molecular dynamics (MD) simulations have been developed to simulate systems where an explicit description of changes in the electronic structure allows for chemical reaction. However, using QM/MM MD simulations is computationally expensive compared to fully classical simulations as a large region (tens of atoms) need to be treated at the Density Functional Theory (DFT) level or respective openshell wave-function based method, to describe accurately radical reactions in the QM part. Quite interestingly, approaches have been proposed recently to replace the QM description with machine-learned (ML) models providing for a tremendous gain in computational efficiency (see for instance [112-115]). Accordingly, we expect that soon such methods will be tuned to allow for carrying out simulations that account for the whole set of chemical reactions taking place when model bilayers are exposed to an electric field.

The relevance of these results is not restricted to electroporation-based treatments. Damage of the same topology and characteristics might be encountered whenever cells are exposed to extensive intracellular or extracellular ROS like in ferroptosis or in biomedical applications such as photodynamic (PD) cold atmospheric plasma (CAP) and ultrasound (US) based therapies.

\section{Funding sources}

This work was partially supported by the National Centre for Research and Development, Poland under POWR.03.02.00-00-I003/16 (N.S.). M.T. acknowledges the support from the Contrat État Plan Region Lorraine 2015-2020 subproject MatDS.

\section{Acknowledgments}

The authors acknowledge the Centre de Calcul Régional ROMEO (https://romeo.univreims.fr/) for providing substantial computational resources.The authors thank Paulina Rozborska and Kinga Woźniak for the preliminary preparation of the patch systems and Lea Rems for assistance in the analyses of the free energy calculations.

\section{Declaration of interest statement}


No potential conflict of interest was reported by the authors.

\section{References}

[1] J. Gehl, Electroporation: Theory and methods, perspectives for drug delivery, gene therapy and research, Acta Physiol. Scand. 177 (2003) 437-447. https://doi.org/10.1046/j.1365-201X.2003.01093.x.

[2] T.S. Anirudhan, S.S. Nair, Development of voltage gated transdermal drug delivery platform to impose synergistic enhancement in skin permeation using electroporation and gold nanoparticle, Mater. Sci. Eng. C. 102 (2019) 437-446. https://doi.org/10.1016/j.msec.2019.04.044.

[3] M.L. Yarmush, A. Golberg, G. Serša, T. Kotnik, D. Miklavčič, Electroporation-Based Technologies for Medicine: Principles, Applications, and Challenges, Annu. Rev. Biomed. Eng. 16 (2014) 295-320. https://doi.org/10.1146/annurev-bioeng-071813104622.

[4] T. Kotnik, L. Rems, M. Tarek, D. Miklavčič, Membrane Electroporation and Electropermeabilization: Mechanisms and Models, Annu. Rev. Biophys. 48 (2019) 63-91. https://doi.org/10.1146/annurev-biophys-052118-115451.

[5] L. Gibot, A. Montigny, H. Baaziz, I. Fourquaux, M. Audebert, M.-P.P. Rols, Calcium Delivery by Electroporation Induces In Vitro Cell Death through Mitochondrial Dysfunction without DNA Damages., Cancers (Basel). 12 (2020) 425. https://doi.org/10.3390/cancers 12020425.

[6] L. Pasquet, S. Chabot, E. Bellard, M.P. Rols, J. Teissie, M. Golzio, Noninvasive Gene Electrotransfer in Skin, Hum. Gene Ther. Methods. 30 (2019) 17-22. https://doi.org/10.1089/hgtb.2018.051.

[7] M. Casciola, M. Tarek, A molecular insight into the electro-transfer of small molecules through electropores driven by electric fields, Biochim. Biophys. Acta - Biomembr. 1858 (2016) 2278-2289. https://doi.org/10.1016/j.bbamem.2016.03.022.

[8] W. Bo, M. Silkunas, U. Mangalanathan, V. Novickij, M. Casciola, I. Semenov, S. Xiao, O.N. Pakhomova, A.G. Pakhomov, Probing Nanoelectroporation and Resealing of the Cell Membrane by the Entry of Ca2+ and Ba2+ Ions, Int. J. Mol. Sci. 21 (2020) 3386. https://doi.org/10.3390/ijms21093386.

[9] J. TEISSIE, M.-P. ROLS, Manipulation of Cell Cytoskeleton Affects the Lifetime of Cell Membrane Electropermeabilization, Ann. N. Y. Acad. Sci. 720 (1994) 98-110. https://doi.org/10.1111/j.1749-6632.1994.tb30438.x.

[10] A.G. Pakhomov, A.M. Bowman, B.L. Ibey, F.M. Andre, O.N. Pakhomova, K.H. Schoenbach, Lipid nanopores can form a stable, ion channel-like conduction pathway in cell membrane, Biochem. Biophys. Res. Commun. 385 (2009) 181-186. https://doi.org/10.1016/j.bbrc.2009.05.035.

[11] M.P. Rols, J. Teissié, Electropermeabilization of mammalian cells. Quantitative analysis of the phenomenon., Biophys. J. 58 (1990) 1089-1098. https://doi.org/10.1016/S0006-3495(90)82451-6.

[12] H. Hanna, A. Denzi, M. Liberti, F.M. André, L.M. Mir, Electropermeabilization of Inner and Outer Cell Membranes with Microsecond Pulsed Electric Fields: Quantitative Study with Calcium Ions, Sci. Rep. 7 (2017) 1-14. https://doi.org/10.1038/s41598-017-12960-w.

[13] Z.A. Levine, P.T. Vernier, Life cycle of an electropore: Field-dependent and field- 
independent steps in pore creation and annihilation, J. Membr. Biol. 236 (2010) 27-36. https://doi.org/10.1007/s00232-010-9277-y.

[14] W.F.D. Bennett, N. Sapay, D.P. Tieleman, Atomistic simulations of pore formation and closure in lipid bilayers, Biophys. J. 106 (2014) 210-219.

https://doi.org/10.1016/j.bpj.2013.11.4486.

[15] L.C. Benov, P.A. Antonov, S.R. Ribarov, Oxidative damage of the membrane-lipids after electroporation, Gen. Physiol. Biophys. 13 (1994) 85-97.

[16] B. Gabriel, J. Teissie, Generation of reactive-oxygen species induced by electropermeabilization of Chinese hamster ovary cells and their consequence on cell viability, Eur. J. Biochem. 223 (1994) 25-33. https://doi.org/10.1111/j.14321033.1994.tb18962.x.

[17] M. Maccarrone, M.R. Bladergroen, N. Rosato, A.F. Agro, Role of Lipid Peroxidation in Electroporation-Induced Cell Permeability, Biochem. Biophys. Res. Commun. 209 (1995) 417-425. https://doi.org/10.1006/bbrc.1995.1519.

[18] M. Maccarrone, N. Rosato, A.F. Agrò, Electroporation enhances cell membrane peroxidation and luminescence., Biochem. Biophys. Res. Commun. 206 (1995) 238245. https://doi.org/10.1006/bbrc.1995.1033.

[19] Y. Zhou, C.K. Berry, P.A. Storer, R.M. Raphael, Peroxidation of polyunsaturated phosphatidyl-choline lipids during electroformation, Biomaterials. 28 (2007) 12981306. https://doi.org/10.1016/j.biomaterials.2006.10.016.

[20] O.N. Pakhomova, V.A. Khorokhorina, A.M. Bowman, R. Rodaitè-Riševičienè, G. Saulis, S. Xiao, A.G. Pakhomov, Oxidative effects of nanosecond pulsed electric field exposure in cells and cell-free media., Arch. Biochem. Biophys. 527 (2012) 55-64. https://doi.org/10.1016/j.abb.2012.08.004.

[21] M. Breton, M. Amirkavei, L.M. Mir, Optimization of the Electroformation of Giant Unilamellar Vesicles (GUVs) with Unsaturated Phospholipids, J. Membr. Biol. 248 (2015) 827-835. https://doi.org/10.1007/s00232-015-9828-3.

[22] S.-K. Yeo, M.-T. Liong, Effect of electroporation on viability and bioconversion of isoflavones in mannitol-soymilk fermented by lactobacilli and bifidobacteria, J. Sci. Food Agric. 93 (2013) 396-409. https://doi.org/10.1002/jsfa.5775.

[23] O. Yun, X.-A. Zeng, C. Brennan, Z. Han, Effect of Pulsed Electric Field on Membrane Lipids and Oxidative Injury of Salmonella typhimurium, Int. J. Mol. Sci. 17 (2016) 1374. https://doi.org/10.3390/ijms17081374.

[24] U. Biedinger, R.J. Youngman, H. Schnabl, Differential effects of electrofusion and electropermeabilization parameters on the membrane integrity of plant protoplasts, Planta. 180 (1990) 598-602. https://doi.org/10.1007/BF02411459.

[25] P.T. Vernier, Z.A. Levine, Y.-H. Wu, V. Joubert, M.J. Ziegler, L.M. Mir, D.P. Tieleman, Electroporating Fields Target Oxidatively Damaged Areas in the Cell Membrane, PLoS One. 4 (2009) e7966. https://doi.org/10.1371/journal.pone.0007966.

[26] S. Kalghatgi, C.S. Spina, J.C. Costello, M. Liesa, J.R. Morones-Ramirez, S. Slomovic, A. Molina, O.S. Shirihai, J.J. Collins, Bactericidal antibiotics induce mitochondrial dysfunction and oxidative damage in mammalian cells, Sci. Transl. Med. 5 (2013) 192ra85-192ra85. https://doi.org/10.1126/scitranslmed.3006055.

[27] B. GABRIEL, J. TEISSIE, Generation of reactive-oxygen species induced by electropermeabilization of Chinese hamster ovary cells and their consequence on cell viability, Eur. J. Biochem. 223 (1994) 25-33. https://doi.org/10.1111/j.14321033.1994.tb18962.x.

[28] P. Jurkiewicz, A. Olzyńska, L. Cwiklik, E. Conte, P. Jungwirth, F.M. Megli, M. Hof, Biophysics of lipid bilayers containing oxidatively modified phospholipids: Insights from fluorescence and EPR experiments and from MD simulations, Biochim. Biophys. 
Acta - Biomembr. 1818 (2012) 2388-2402.

https://doi.org/10.1016/j.bbamem.2012.05.020.

[29] L. Beranova, L. Cwiklik, P. Jurkiewicz, M. Hof, P. Jungwirth, Oxidation changes physical properties of phospholipid bilayers: Fluorescence spectroscopy and molecular simulations, Langmuir. 26 (2010) 6140-6144. https://doi.org/10.1021/la100657a.

[30] M.P. Rols, J. Teissié, Experimental evidence for the involvement of the cytoskeleton in mammalian cell electropermeabilization, BBA - Biomembr. 1111 (1992) 45-50. https://doi.org/10.1016/0005-2736(92)90272-N.

[31] W. Ren, N.M. Sain, S.J. Beebe, Nanosecond pulsed electric fields (nsPEFs) activate intrinsic caspase-dependent and caspase-independent cell death in Jurkat cells, Biochem. Biophys. Res. Commun. 421 (2012) 808-812. https://doi.org/10.1016/j.bbrc.2012.04.094.

[32] P. Bonnafous, M.C. Vernhes, J. Teissié, B. Gabriel, The generation of reactive-oxygen species associated with long-lasting pulse-induced electropermeabilisation of mammalian cells is based on a non-destructive alteration of the plasma membrane, Biochim. Biophys. Acta - Biomembr. 1461 (1999) 123-134. https://doi.org/10.1016/S0005-2736(99)00154-6.

[33] M. Breton, L.M. Mir, Investigation of the chemical mechanisms involved in the electropulsation of membranes at the molecular level, Bioelectrochemistry. 119 (2018) 76-83. https://doi.org/10.1016/j.bioelechem.2017.09.005.

[34] M.M. Gaschler, B.R. Stockwell, Lipid peroxidation in cell death, Biochem. Biophys. Res. Commun. 482 (2017) 419-425. https://doi.org/10.1016/j.bbrc.2016.10.086.

[35] R. Nuccitelli, K. Lui, M. Kreis, B. Athos, P. Nuccitelli, Nanosecond pulsed electric field stimulation of reactive oxygen species in human pancreatic cancer cells is $\mathrm{Ca} 2+-$ dependent, Biochem. Biophys. Res. Commun. 435 (2013) 580-585. https://doi.org/10.1016/j.bbrc.2013.05.014.

[36] L.C. Benov, P.A. Antonov, S.R. Ribarov, Oxidative damage of the membrane lipids after electroporation., Gen. Physiol. Biophys. 13 (1994) 85-97.

[37] N. Sabri, B. Pelissier, J. Teissie, Electropermeabilization of Intact Maize Cells Induces an Oxidative Stress, Eur. J. Biochem. 238 (1996) 737-743. https://doi.org/10.1111/j.1432-1033.1996.0737w.x.

[38] R.M. Cordeiro, Reactive oxygen species at phospholipid bilayers: Distribution, mobility and permeation, Biochim. Biophys. Acta - Biomembr. 1838 (2014) 438-444. https://doi.org/10.1016/j.bbamem.2013.09.016.

[39] G. Weber, T. Charitat, M.S. Baptista, A.F. Uchoa, C. Pavani, H.C. Junqueira, Y. Guo, V.A. Baulin, R. Itri, C.M. Marques, A.P. Schroder, Lipid oxidation induces structural changes in biomimetic membranes, Soft Matter. 10 (2014) 4241-4247. https://doi.org/10.1039/c3sm52740a.

[40] K.A. Runas, N. Malmstadt, Low levels of lipid oxidation radically increase the passive permeability of lipid bilayers, Soft Matter. 11 (2015) 499-505. https://doi.org/10.1039/c4sm01478b.

[41] K.M. Schaich, J. Xie, B.A. Bogusz, Thinking outside the classical chain reaction box of lipid oxidation: Evidence for alternate pathways and the importance of epoxides, Lipid Technol. 29 (2017) 91-96. https://doi.org/10.1002/lite.201700025.

[42] G. Spiteller, M. Afzal, The Action of Peroxyl Radicals, Powerful Deleterious Reagents, Explains Why Neither Cholesterol Nor Saturated Fatty Acids Cause Atherogenesis and Age-Related Diseases, Chem. - A Eur. J. 20 (2014) 14928-14945. https://doi.org/10.1002/chem.201404383.

[43] Z.A.M. Zielinski, D.A. Pratt, Lipid Peroxidation: Kinetics, Mechanisms, and Products, J. Org. Chem. 82 (2017) 2817-2825. https://doi.org/10.1021/acs.joc.7b00152. 
[44] A. Reis, C.M. Spickett, Chemistry of phospholipid oxidation, Biochim. Biophys. Acta - Biomembr. 1818 (2012) 2374-2387. https://doi.org/10.1016/j.bbamem.2012.02.002.

[45] R. Domínguez, M. Pateiro, M. Gagaoua, F.J. Barba, W. Zhang, J.M. Lorenzo, A Comprehensive Review on Lipid Oxidation in Meat and Meat Products, Antioxidants. 8 (2019) 429. https://doi.org/10.3390/antiox8100429.

[46] L. Rems, M. Viano, M.A. Kasimova, D. Miklavčič, M. Tarek, The contribution of lipid peroxidation to membrane permeability in electropermeabilization: A molecular dynamics study, Bioelectrochemistry. 125 (2019) 46-57. https://doi.org/10.1016/j.bioelechem.2018.07.018.

[47] K.A. Riske, T.P. Sudbrack, N.L. Archilha, A.F. Uchoa, A.P. Schroder, C.M. Marques, M.S. Baptista, R. Itri, Giant vesicles under oxidative stress induced by a membraneanchored photosensitizer, Biophys. J. 97 (2009) 1362-1370. https://doi.org/10.1016/j.bpj.2009.06.023.

[48] G.O. Fruhwirth, A. Loidl, A. Hermetter, Oxidized phospholipids: From molecular properties to disease, Biochim. Biophys. Acta - Mol. Basis Dis. 1772 (2007) 718-736. https://doi.org/10.1016/j.bbadis.2007.04.009.

[49] C.C. Lai, S.H. Yang, B.J. Finlayson-Pitts, Interactions of Monolayers of Unsaturated Phosphocholines with Ozone at the Air-Water Interface, Langmuir. 10 (1994) 46374644. https://doi.org/10.1021/la00024a041.

[50] P. Boonnoy, V. Jarerattanachat, M. Karttunen, J. Wong-Ekkabut, Bilayer Deformation, Pores, and Micellation Induced by Oxidized Lipids, J. Phys. Chem. Lett. 6 (2015) 4884-4888. https://doi.org/10.1021/acs.jpclett.5b02405.

[51] J. Wong-Ekkabut, Z. Xu, W. Triampo, I.M. Tang, D.P. Tieleman, L. Monticelli, Effect of lipid peroxidation on the properties of lipid bilayers: A molecular dynamics study, Biophys. J. 93 (2007) 4225-4236. https://doi.org/10.1529/biophysj.107.112565.

[52] S. Sankhagowit, S.H. Wu, R. Biswas, C.T. Riche, M.L. Povinelli, N. Malmstadt, The dynamics of giant unilamellar vesicle oxidation probed by morphological transitions, Biochim. Biophys. Acta - Biomembr. 1838 (2014) 2615-2624. https://doi.org/10.1016/j.bbamem.2014.06.020.

[53] T.M. Tsubone, M.S. Baptista, R. Itri, Understanding membrane remodelling initiated by photosensitized lipid oxidation, Biophys. Chem. 254 (2019) 106263. https://doi.org/10.1016/j.bpc.2019.106263.

[54] M.A. Cuendet, M.E. Tuckerman, Free energy reconstruction from metadynamics or adiabatic free energy dynamics simulations, J. Chem. Theory Comput. 10 (2014) 2975-2986. https://doi.org/10.1021/ct500012b.

[55] J.B. Klauda, R.M. Venable, J.A. Freites, J.W. O’Connor, D.J. Tobias, C. MondragonRamirez, I. Vorobyov, A.D. MacKerell, R.W. Pastor, Update of the CHARMM AllAtom Additive Force Field for Lipids: Validation on Six Lipid Types, J. Phys. Chem. B. 114 (2010) 7830-7843. https://doi.org/10.1021/jp101759q.

[56] W.L. Jorgensen, J. Chandrasekhar, J.D. Madura, R.W. Impey, M.L. Klein, Comparison of simple potential functions for simulating liquid water, J. Chem. Phys. 79 (1983) 926-935. https://doi.org/10.1063/1.445869.

[57] J. Melcr, H. Martinez-Seara, R. Nencini, J. Kolafa, P. Jungwirth, O.H.S. Ollila, Accurate Binding of Sodium and Calcium to a POPC Bilayer by Effective Inclusion of Electronic Polarization, J. Phys. Chem. B. 122 (2018) 4546-4557. https://doi.org/10.1021/acs.jpcb.7b12510.

[58] M.J. Abraham, T. Murtola, R. Schulz, S. Páll, J.C. Smith, B. Hess, E. Lindah, Gromacs: High performance molecular simulations through multi-level parallelism from laptops to supercomputers, SoftwareX. 1-2 (2015) 19-25. https://doi.org/10.1016/j.softx.2015.06.001. 
[59] D.J. Evans, B.L. Holian, The Nose-Hoover thermostat, J. Chem. Phys. 83 (1985) 4069-4074. https://doi.org/10.1063/1.449071.

[60] M. Parrinello, A. Rahman, Polymorphic transitions in single crystals: A new molecular dynamics method, J. Appl. Phys. 52 (1981) 7182-7190. https://doi.org/10.1063/1.328693.

[61] T. Darden, D. York, L. Pedersen, Particle mesh Ewald: An N· $\log (\mathrm{N})$ method for Ewald sums in large systems, J. Chem. Phys. 98 (1993) 10089-10092. https://doi.org/10.1063/1.464397.

[62] B.A. Wilson, A. Ramanathan, C.F. Lopez, LoLab-VU/PyBILT: A Python toolkit to analyze molecular simulation trajectories of lipid bilayer systems., (2019). https://doi.org/10.5281/ZENODO.3426128.

[63] W. Humphrey, A. Dalke, K. Schulten, VMD: Visual molecular dynamics, J. Mol. Graph. 14 (1996) 33-38. https://doi.org/10.1016/0263-7855(96)00018-5.

[64] M. Chen, M.A. Cuendet, M.E. Tuckerman, Heating and flooding: A unified approach for rapid generation of free energy surfaces, J. Chem. Phys. 137 (2012) 024102. https://doi.org/10.1063/1.4733389.

[65] J.M. Escoffre, P. Campomanes, M. Tarek, A. Bouakaz, New insights on the role of ROS in the mechanisms of sonoporation-mediated gene delivery, Ultrason. Sonochem. 64 (2020) 104998. https://doi.org/10.1016/j.ultsonch.2020.104998.

[66] D. Bonhenry, F. Dehez, M. Tarek, Effects of hydration on the protonation state of a lysine analog crossing a phospholipid bilayer-insights from molecular dynamics and free-energy calculations, Phys. Chem. Chem. Phys. 20 (2018) 9101-9107. https://doi.org/10.1039/c8cp00312b.

[67] B. Hess, C. Kutzner, D. Van Der Spoel, E. Lindahl, GRGMACS 4: Algorithms for highly efficient, load-balanced, and scalable molecular simulation, J. Chem. Theory Comput. 4 (2008) 435-447. https://doi.org/10.1021/ct700301q.

[68] M. Bonomi, D. Branduardi, G. Bussi, C. Camilloni, D. Provasi, P. Raiteri, D. Donadio, F. Marinelli, F. Pietrucci, R.A. Broglia, M. Parrinello, PLUMED: A portable plugin for free-energy calculations with molecular dynamics, Comput. Phys. Commun. 180 (2009) 1961-1972. https://doi.org/10.1016/j.cpc.2009.05.011.

[69] B. Hess, H. Bekker, H.J.C. Berendsen, J.G.E.M. Fraaije, LINCS: A linear constraint solver for molecular simulations, J. Comput. Chem. 18 (1997) 1463-1472. https://doi.org/10.1002/(SICI)1096-987X(199709)18:12<1463::AID-JCC4>3.0.CO;2H.

[70] I. Vorobyov, T.E. Olson, J.H. Kim, R.E. Koeppe, O.S. Andersen, T.W. Allen, Ioninduced defect permeation of lipid membranes, Biophys. J. 106 (2014) 586-597. https://doi.org/10.1016/j.bpj.2013.12.027.

[71] O.S. Andersen, M. Fuchs, Potential energy barriers to ion transport within lipid bilayers. Studies with tetraphenylborate., Biophys. J. 15 (1975) 795-830. https://doi.org/10.1016/S0006-3495(75)85856-5.

[72] G. Hummer, Position-dependent diffusion coefficients and free energies from Bayesian analysis of equilibrium and replica molecular dynamics simulations, New J. Phys. 7 (2005) 34. https://doi.org/10.1088/1367-2630/7/1/034.

[73] J.D. Watson, F.H.C. Crick, Molecular structure of nucleic acids: A structure for deoxyribose nucleic acid, Nature. 171 (1953) 737-738. https://doi.org/10.1038/171737a0.

[74] J.L. Young, D.A. Dean, Electroporation-Mediated Gene Delivery, Adv. Genet. 89 (2015) 49-88. https://doi.org/10.1016/bs.adgen.2014.10.003.

[75] H. Saito, T. Morishita, T. Mizukami, K.I. Nishiyama, K. Kawaguchi, H. Nagao, Molecular dynamics study of binary POPC bilayers: Molecular condensing effects on 
membrane structure and dynamics, in: J. Phys. Conf. Ser., Institute of Physics Publishing, 2018: p. 12022. https://doi.org/10.1088/1742-6596/1136/1/012022.

[76] A. Filippov, G. Orädd, G. Lindblom, Influence of cholesterol and water content on phospholipid lateral diffusion in bilayers, Langmuir. 19 (2003) 6397-6400. https://doi.org/10.1021/la034222x.

[77] T.Y. Wang, M.D.J. Libardo, A.M. Angeles-Boza, J.P. Pellois, Membrane Oxidation in Cell Delivery and Cell Killing Applications, ACS Chem. Biol. 12 (2017) 1170-1182. https://doi.org/10.1021/acschembio.7b00237.

[78] C. Mu, J. Wang, K.M. Barraza, X. Zhang, J.L. Beauchamp, Mass Spectrometric Study of Acoustically Levitated Droplets Illuminates Molecular- Level Mechanism of Photodynamic Therapy for Cancer involving Lipid Oxidation, Angew. Chemie Int. Ed 58 (2019) 8082-8086. https://doi.org/10.1002/anie.201902815.

[79] J. Garrec, A. Monari, X. Assfeld, L.M. Mir, M. Tarek, Lipid peroxidation in membranes: The peroxyl radical does not "float," J. Phys. Chem. Lett. 5 (2014) 16531658. https://doi.org/10.1021/jz500502q.

[80] J. Van Der Paal, E.C. Neyts, C.C.W. Verlackt, A. Bogaerts, Effect of lipid peroxidation on membrane permeability of cancer and normal cells subjected to oxidative stress, Chem. Sci. 7 (2016) 489-498. https://doi.org/10.1039/c5sc02311d.

[81] M. Pavlin, V. Leben, D. Miklavčič, Electroporation in dense cell suspensionTheoretical and experimental analysis of ion diffusion and cell permeabilization, Biochim. Biophys. Acta - Gen. Subj. 1770 (2007) 12-23. https://doi.org/10.1016/j.bbagen.2006.06.014.

[82] J. Nourooz-Zadeh, Ferrous ion oxidation in presence of xylenol orange for detection of lipid hydroperoxides in plasma, Methods Enzymol. 300 (1999) 58-62. https://doi.org/10.1016/S0076-6879(99)00113-5.

[83] C.W. Kwon, K.-M. Park, J.W. Park, J. Lee, S.J. Choi, P.-S. Chang, Rapid and Sensitive Determination of Lipid Oxidation Using the Reagent Kit Based on Spectrophotometry (FOODLAB fat System), J. Chem. 2016 (2016) 1-6. https://doi.org/10.1155/2016/1468743.

[84] J. Nourooz-Zadeh, N.K. Gopaul, S. Barrow, A.I. Mallet, E.E. Änggård, Analysis of F2-isoprostanes as indicators of non-enzymatic lipid peroxidation in vivo by gas chromatography-mass spectrometry: development of a solid-phase extraction procedure, J. Chromatogr. B Biomed. Sci. Appl. 667 (1995) 199-208. https://doi.org/10.1016/0378-4347(95)00035-H.

[85] A. Bachi, E. Zuccato, M. Baraldi, R. Fanelli, C. Chiabrando, Measurement of urinary 8 -epi-prostaglandin F2 $\alpha$, a novel index of lipid peroxidation in vivo, by immunoaffinity extraction/gas chromatography-mass spectrometry. Basal levels in smokers and nonsmokers, Free Radic. Biol. Med. 20 (1996) 619-624. https://doi.org/10.1016/0891-5849(95)02087-X.

[86] L.A. Voloboueva, J. Liu, J.H. Suh, B.N. Ames, S.S. Miller, (R)- $\alpha$-lipoic acid protects retinal pigment epithelial cells from oxidative damage, Investig. Ophthalmol. Vis. Sci. 46 (2005) 4302-4310. https://doi.org/10.1167/iovs.04-1098.

[87] O. Michel, A.G. Pakhomov, M. Casciola, J. Saczko, J. Kulbacka, O.N. Pakhomova, Electropermeabilization does not correlate with plasma membrane lipid oxidation, Bioelectrochemistry. 132 (2020) 107433. https://doi.org/10.1016/j.bioelechem.2019.107433.

[88] J. Li, H. Lin, The current-voltage relation for electropores with conductivity gradients, Biomicrofluidics. 4 (2010). https://doi.org/10.1063/1.3324847.

[89] A.G. Pakhomov, R. Shevin, J.A. White, J.F. Kolb, O.N. Pakhomova, R.P. Joshi, K.H. Schoenbach, Membrane permeabilization and cell damage by ultrashort electric field 
shocks, Arch. Biochem. Biophys. 465 (2007) 109-118.

https://doi.org/10.1016/j.abb.2007.05.003.

[90] B.L. Ibey, S. Xiao, K.H. Schoenbach, M.R. Murphy, A.G. Pakhomov, Plasma membrane permeabilization by 60- and 600-ns electric pulses is determined by the absorbed dose, Bioelectromagnetics. 30 (2009) 92-99.

https://doi.org/10.1002/bem.20451.

[91] B.L. Ibey, D.G. Mixon, J.A. Payne, A. Bowman, K. Sickendick, G.J. Wilmink, W.P. Roach, A.G. Pakhomov, Plasma membrane permeabilization by trains of ultrashort electric pulses, Bioelectrochemistry. 79 (2010) 114-121.

https://doi.org/10.1016/j.bioelechem.2010.01.001.

[92] O.G. Mouritsen, M.J. Zuckermann, What's so special about cholesterol?, Lipids. 39 (2004) 1101-1113. https://doi.org/10.1007/s11745-004-1336-x.

[93] A.D. Dupuy, D.M. Engelman, Protein area occupancy at the center of the red blood cell membrane, Proc. Natl. Acad. Sci. U. S. A. 105 (2008) 2848-2852. https://doi.org/10.1073/pnas.0712379105.

[94] M.R. Krause, S.L. Regen, The structural role of cholesterol in cell membranes: From condensed bilayers to lipid rafts, Acc. Chem. Res. 47 (2014) 3512-3521. https://doi.org/10.1021/ar500260t.

[95] S. Ramadurai, A. Holt, V. Krasnikov, G. Van Den Bogaart, J.A. Killian, B. Poolman, Lateral diffusion of membrane proteins, J. Am. Chem. Soc. 131 (2009) 12650-12656. https://doi.org/10.1021/ja902853g.

[96] M. Olšinová, P. Jurkiewicz, I. Kishko, J. Sýkora, J. Sabó, M. Hof, L. Cwiklik, M. Cebecauer, Roughness of Transmembrane Helices Reduces Lipid Membrane Dynamics, IScience. 10 (2018) 87-97. https://doi.org/10.1016/j.isci.2018.11.026.

[97] A. Abe, M. Hiraoka, H. Ohguro, J.J. Tesmer, J.A. Shayman, Preferential hydrolysis of truncated oxidized glycerophospholipids by lysosomal phospholipase A2, J. Lipid Res. 58 (2017) 339-349. https://doi.org/10.1194/jlr.M070730.

[98] H. Li, B. Benipal, S. Zhou, C. Dodia, S. Chatterjee, J.Q. Tao, E.M. Sorokina, T. Raabe, S.I. Feinstein, A.B. Fisher, Critical role of peroxiredoxin 6 in the repair of peroxidized cell membranes following oxidative stress, Free Radic. Biol. Med. 87 (2015) 356-365. https://doi.org/10.1016/j.freeradbiomed.2015.06.009.

[99] M. Keidar, ed., Plasma Cancer Therapy, Springer International Publishing, Cham, 2020. https://doi.org/10.1007/978-3-030-49966-2.

[100] M. Yusupov, K. Wende, S. Kupsch, E.C. Neyts, S. Reuter, A. Bogaerts, Effect of head group and lipid tail oxidation in the cell membrane revealed through integrated simulations and experiments, Sci. Rep. 7 (2017) 1-14. https://doi.org/10.1038/s41598017-06412-8.

[101] J. Van Der Paal, S.H. Hong, M. Yusupov, N. Gaur, J.S. Oh, R.D. Short, E.J. Szili, A. Bogaerts, How membrane lipids influence plasma delivery of reactive oxygen species into cells and subsequent DNA damage: An experimental and computational study, Phys. Chem. Chem. Phys. 21 (2019) 19327-19341. https://doi.org/10.1039/c9cp03520f.

[102] M. Yusupov, J. Van der Paal, E.C. Neyts, A. Bogaerts, Synergistic effect of electric field and lipid oxidation on the permeability of cell membranes, Biochim. Biophys. Acta - Gen. Subj. 1861 (2017) 839-847. https://doi.org/10.1016/j.bbagen.2017.01.030.

[103] L. Benov, Photodynamic therapy: Current status and future directions, in: Med. Princ. Pract., S. Karger AG, 2015: pp. 14-28. https://doi.org/10.1159/000362416.

[104] I.O.L. Bacellar, M.S. Baptista, Mechanisms of Photosensitized Lipid Oxidation and Membrane Permeabilization, ACS Omega. 4 (2019) 21636-21646. https://doi.org/10.1021/acsomega.9b03244. 
[105] J. Razzokov, M. Yusupov, R.M. Cordeiro, A. Bogaerts, Atomic scale understanding of the permeation of plasma species across native and oxidized membranes, J. Phys. D. Appl. Phys. 51 (2018) 365203. https://doi.org/10.1088/1361-6463/aad524.

[106] B.L. Heckmann, B. Tummers, D.R. Green, Crashing the computer: apoptosis vs. necroptosis in neuroinflammation, Cell Death Differ. 26 (2019) 41-52. https://doi.org/10.1038/s41418-018-0195-3.

[107] M. Riegman, L. Sagie, C. Galed, T. Levin, N. Steinberg, S.J. Dixon, U. Wiesner, M.S. Bradbury, P. Niethammer, A. Zaritsky, M. Overholtzer, Ferroptosis occurs through an osmotic mechanism and propagates independently of cell rupture, Nat. Cell Biol. 22 (2020) 1042-1048. https://doi.org/10.1038/s41556-020-0565-1.

[108] W.S. Yang, K.J. Kim, M.M. Gaschler, M. Patel, M.S. Shchepinov, B.R. Stockwell, Peroxidation of polyunsaturated fatty acids by lipoxygenases drives ferroptosis, Proc. Natl. Acad. Sci. U. S. A. 113 (2016) E4966-E4975. https://doi.org/10.1073/pnas.1603244113.

[109] Y. Xie, W. Hou, X. Song, Y. Yu, J. Huang, X. Sun, R. Kang, D. Tang, Ferroptosis: Process and function, Cell Death Differ. 23 (2016) 369-379. https://doi.org/10.1038/cdd.2015.158.

[110] A.J. Davidson, W. Wood, Igniting the spread of ferroptotic cell death, Nat. Cell Biol. 22 (2020) 1027-1029. https://doi.org/10.1038/s41556-020-0570-4.

[111] L. Pedrera, R.A. Espiritu, U. Ros, A. Schmitt, S. Hailfinger, A.J. García-Sáez, Ferroptotic pores induce $\mathrm{Ca} 2+$ fluxes and ESCRT-III activation to modulate cell death kinetics, BioRxiv. (2019) 867564. https://doi.org/10.1101/867564.

[112] J. Hermann, Z. Schätzle, F. Noé, Deep-neural-network solution of the electronic Schrödinger equation, Nat. Chem. 12 (2020) 891-897. https://doi.org/10.1038/s41557020-0544-y.

[113] S. Naserifar, Y. Chen, S. Kwon, H. Xiao, W.A. Goddard, Artificial Intelligence and QM/MM with a Polarizable Reactive Force Field for Next-Generation Electrocatalysts, Matter. 4 (2021) 195-216. https://doi.org/10.1016/j.matt.2020.11.010.

[114] K.T. Schütt, M. Gastegger, A. Tkatchenko, K.R. Müller, R.J. Maurer, Unifying machine learning and quantum chemistry with a deep neural network for molecular wavefunctions, Nat. Commun. 10 (2019) 1-10. https://doi.org/10.1038/s41467-01912875-2.

[115] L. Böselt, M. Thürlemann, S. Riniker, Machine Learning in QM/MM Molecular Dynamics Simulations of Condensed-Phase Systems, J. Chem. Theory Comput. (2021). https://doi.org/10.1021/acs.jctc.0c01112. 
Appendices:

Supporting Information 


\section{Figures:}

Schematic 1. Pathway for primary and secondary oxidation of a phospholipid (L) containing a single bis-allylic site e.g. linoleic acid, linoleate ester. The primary oxidation is a multistep process leading to the generation of hydroperoxides (and fuel the chain reaction - yellow arrows). The secondary oxidation can occur either directly through Hock fragmentation (blue arrow) with the formation of 2-nonenal [43] or through Hock fragmentation followed by oxidation as in the case of PoxnoPC [44,45]. 2-nonenal can be a hydrogen donor for LOO• (green arrow) and transfer the radical to another lipid initiating yet another chain reaction.

Schematic 2. Representation of oxidized membrane lesions (patches made of secondary oxidized lipids, drawn in purple) expected to be formed in the cell membrane following exposure to electric pulses (Left). The schematic is hypothetical, and the lesions are not drawn to scale. Middle: cartoon of molecular organization in one of the lesions, showing a patch of secondary oxidized lipids. Right: State of the membrane when the secondary oxidized lipids diffused out. Image adapted from [46].

Fig. 1. Time evolution of pore formation and its closure in the bilayer systems without cholesterol (SYST I : top six blue panels) and with cholesterol (SYST II: bottom six orange panels). The grid size is $1 \mathrm{~nm}$. The blue and white spheres represent the choline and phosphate groups of the POPC molecules' zwitterionic head groups. The magenta and black spheres represent the choline and phosphate groups of PoxnoPCs head groups, the red spheres correspond to aldehyde oxygen of PoxnoPCs lipid tails, and the cholesterol's $\mathrm{OH}$ groups are drawn as yellow spheres. The corresponding membrane cross-sections can be found in SI, Figure S2.3.1. and Figure S2.3.2. along with the videos of the full MD trajectories.

Fig. 2. Thickness (A) and average area per lipid (B) for BIL1, BIL2, BIL3, BIL4 lipid membranes. The legend (red $-50 \%$, green $-20 \%$, orange $-10 \%$, blue $-0 \%$ ) reports the percentage of secondary oxidized lipids (PoxnoPC) in POPC bilayer, where $0 \%$ represents data for the pure POPC bilayer. The bars indicate standard deviations based on the last $100 \mathrm{~ns}$ of the MD trajectories.

Fig. 3. The PMF profiles of $\mathrm{Na}^{+}$(A) and $\mathrm{Cl}^{-}(\mathrm{B})$ in BILL1 to BILL4. The legend represents the ration PoxnoPC:POPC and $\mathrm{z}$ the ion position with respect to the center of the bilayer (located at $\mathrm{z}=0$ ). The error bars on the free energy estimates (see 2.2.3) are less than $2 \mathrm{~kJ} / \mathrm{mol}$ (see $\mathrm{S} 3.5$ for details).

Fig. 4. Permeability to $\mathrm{Na}^{+}(\mathrm{A})$ and $\mathrm{Cl}^{-}(\mathrm{B})$ and overall membrane for both ions (C) of the BIL1 - BIL4 defined by the percentage of secondary oxidized lipids (PoxnoPC) in the POPC bilayer, $(0 \%$, corresponding to BIL1 is the pure POPC lipid membrane. 


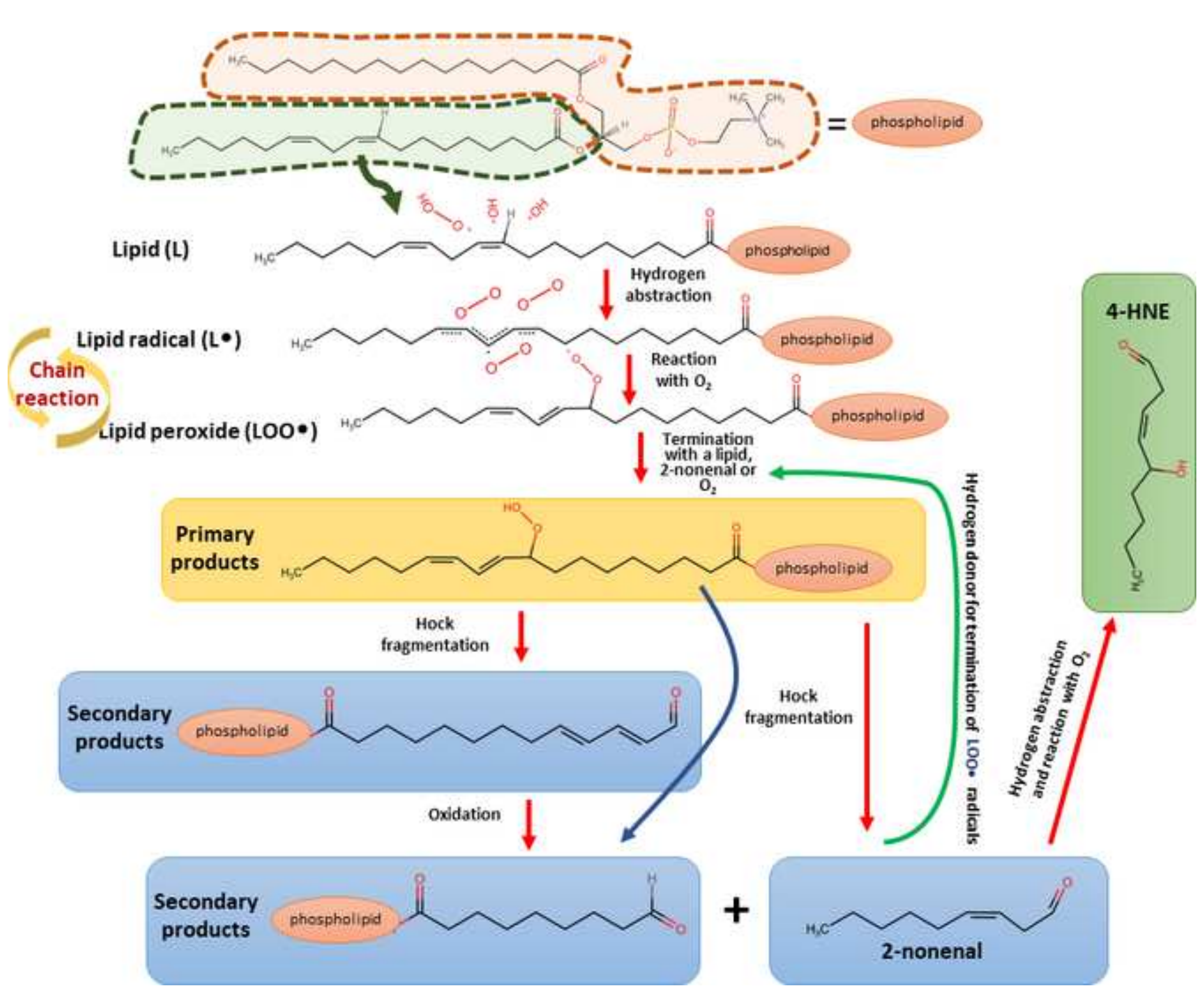




\section{After pulse}
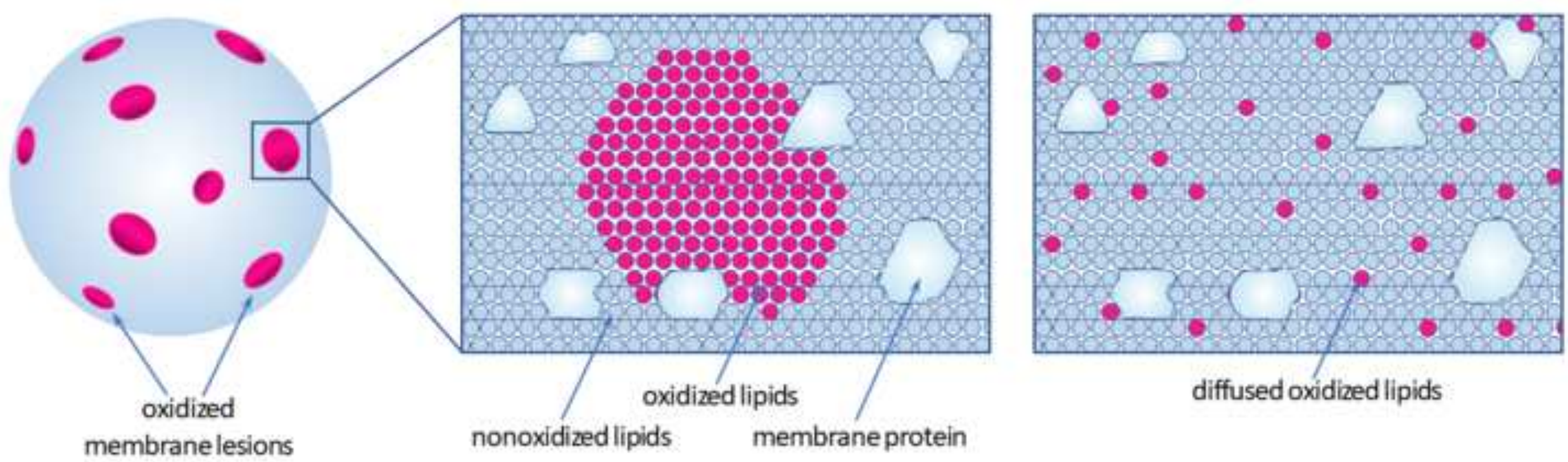

membrane lesions

nonoxidized lipids

membrane protein 

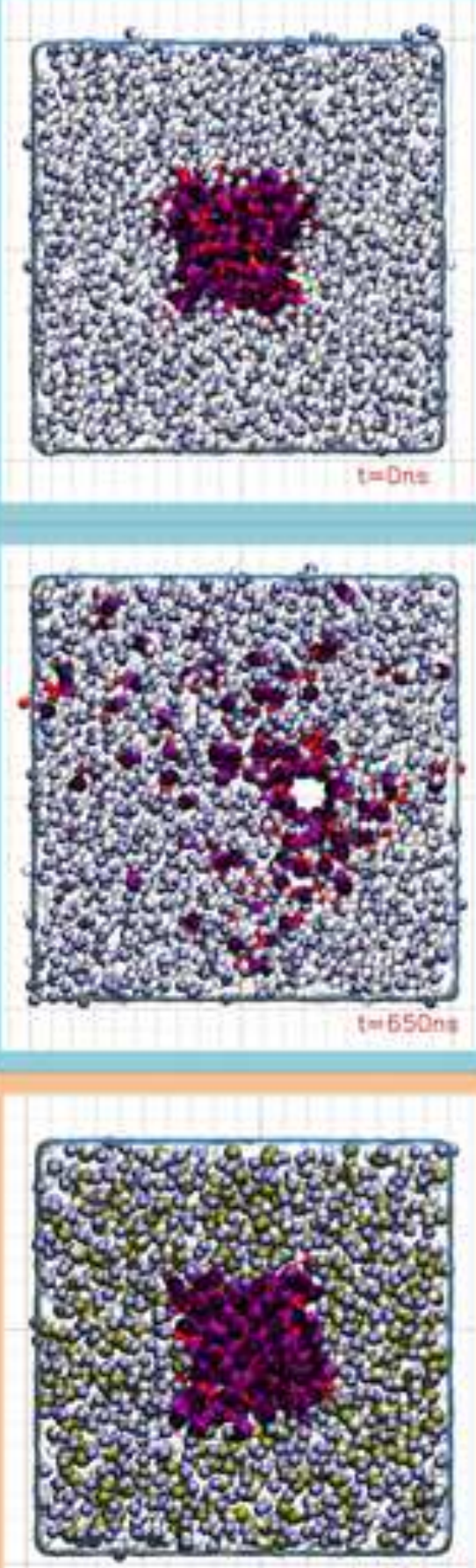

tw0ns

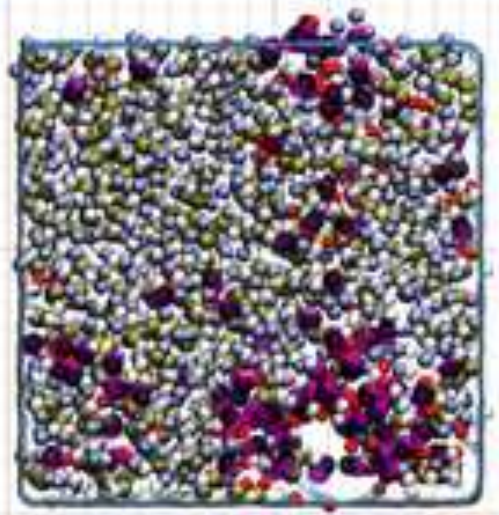

$\mathrm{t}=2500 \mathrm{n}$
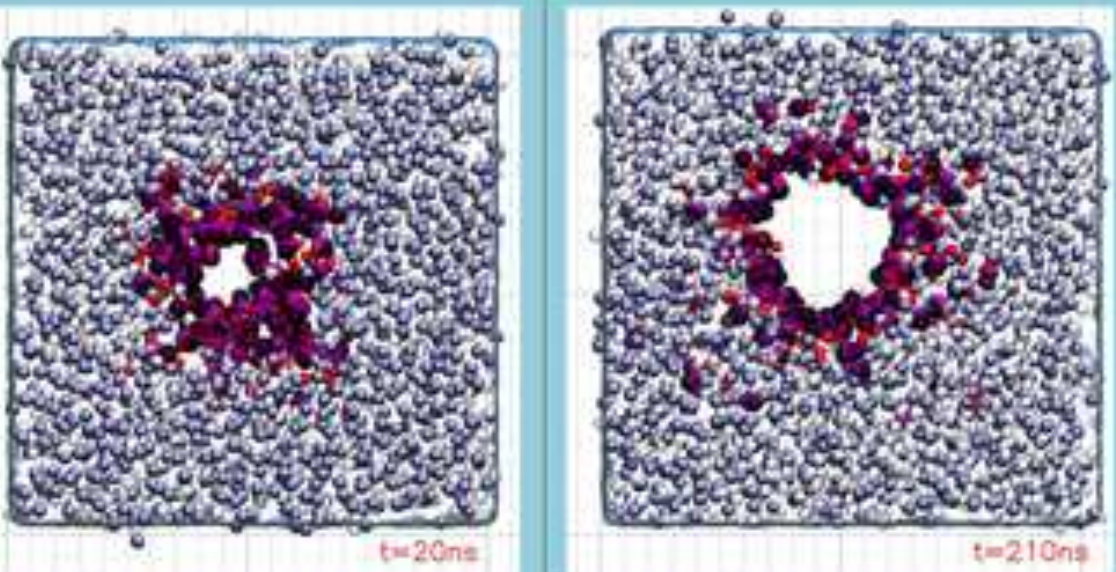

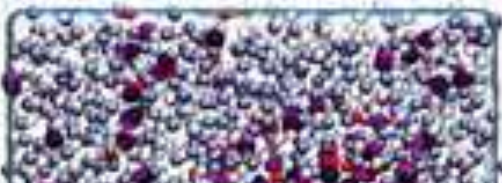

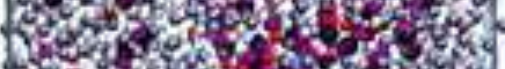

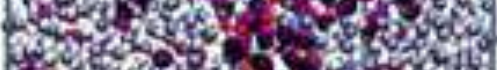

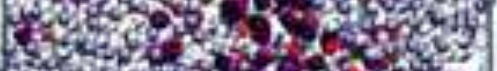

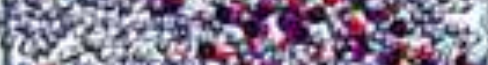

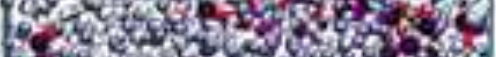

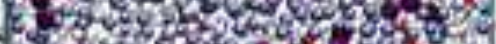

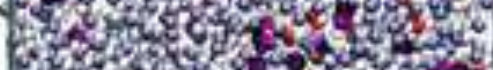

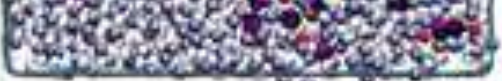
ing $70 n$ is
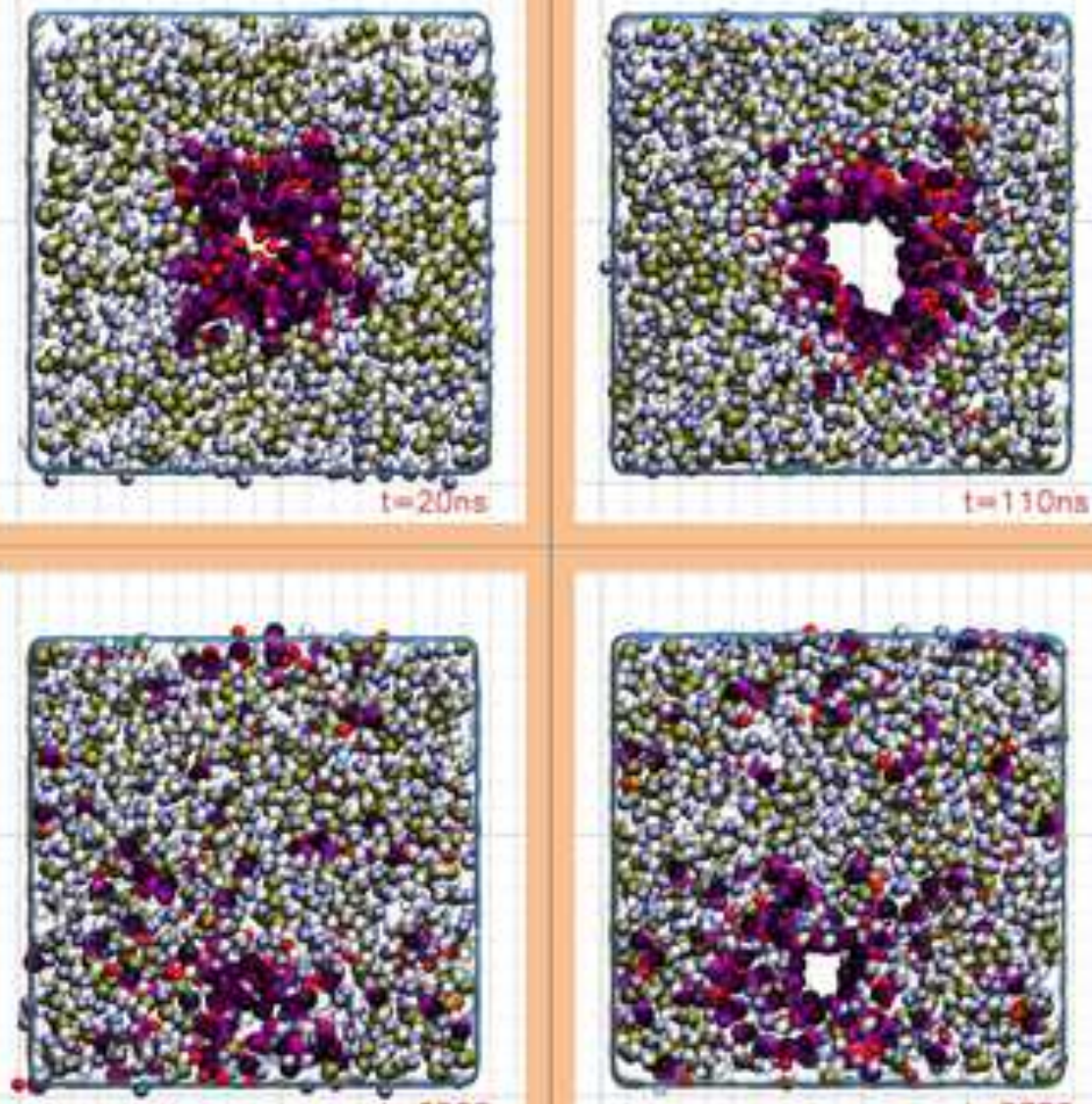

$t=5380 n$ 

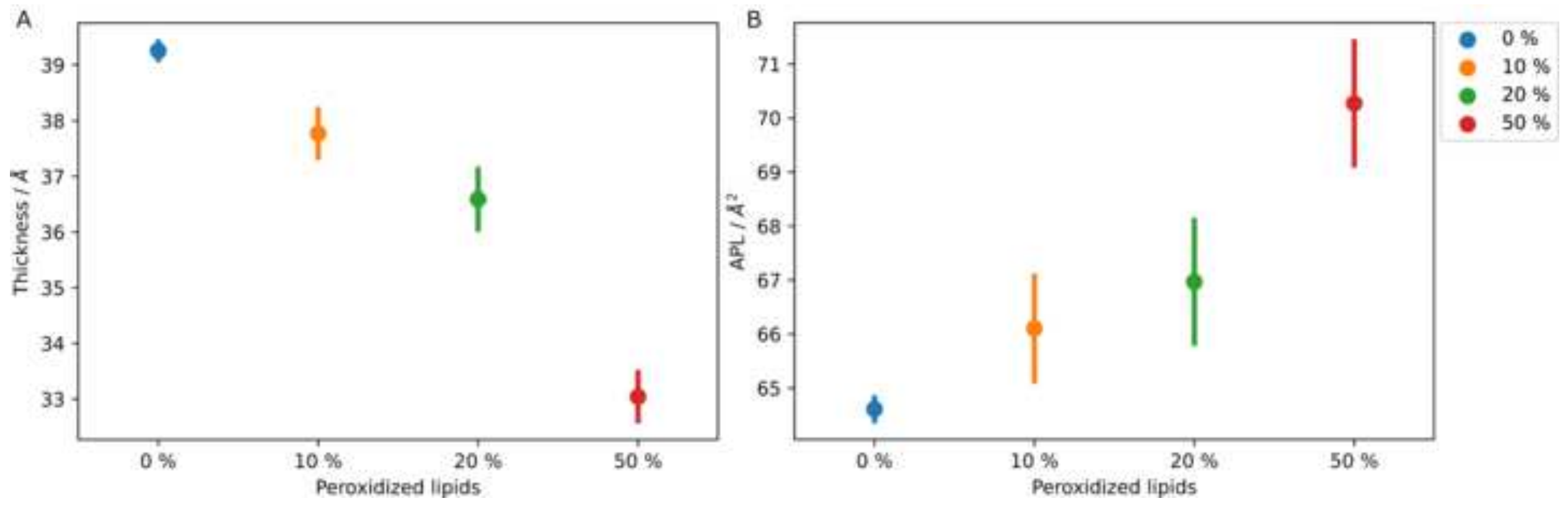

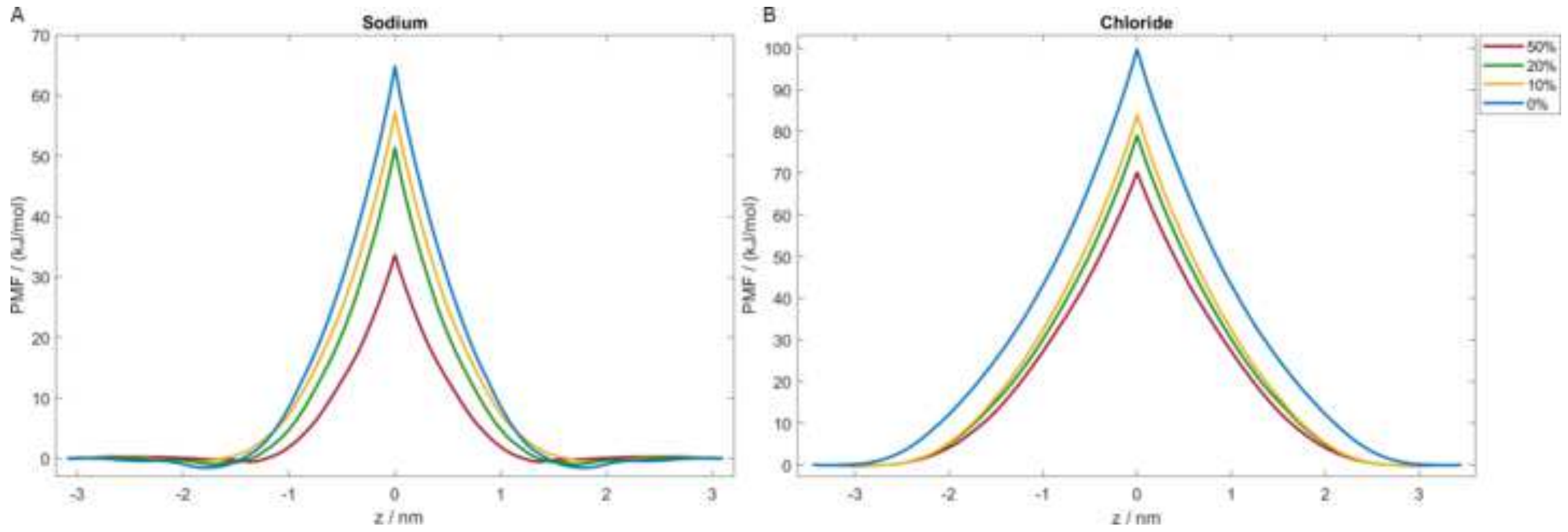

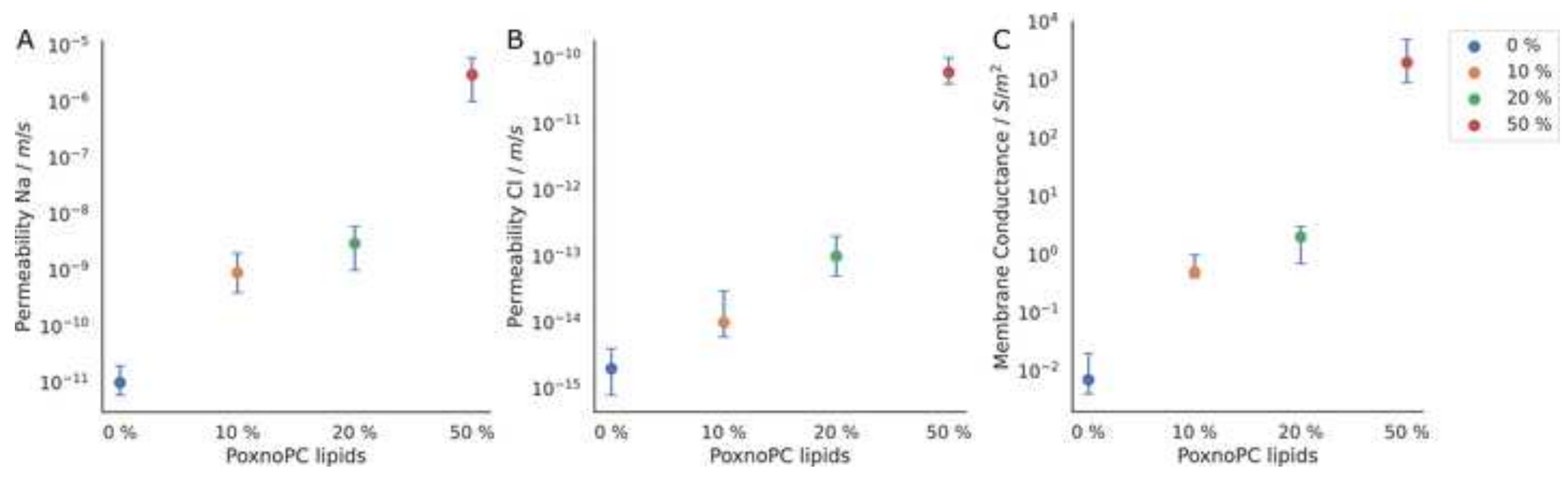